\title{
Emergence of an egocentric cue guiding and allocentric inferring strategy that mirrors hippocampal brain-derived neurotrophic factor (BDNF) expression in the Morris water maze
}

\author{
Deirdre R. Harvey, Anne-Marie T. McGauran, Jonathan Murphy, Lauren Burns, \\ Eoghan McMonagle, Sean Commins *
}

Department of Psychology, National University of Ireland-Maynooth, Maynooth, Co. Kildare, Ireland

Received 20 June 2007; revised 23 August 2007; accepted 24 August 2007

Available online 17 October 2007

\begin{abstract}
From insects to humans, successful navigation relies on retained representations of spatial relations. These representations are thought to depend on the hippocampal formation, particularly those that are independent of the navigator (allocentric representations). The Morris water maze is a simple and popular task often used to assess spatial navigation. But how animals navigate toward and retain information regarding the location of the goal in this task remains unclear. We provide a comprehensive account of how the water maze is accomplished behaviourally. Our findings suggest that animals solve the task using distal cues via an initial view-matching strategy that is supported by egocentric guidance. Through increased training, however, an emergence of an egocentric-guiding strategy combined with the animal's greater ability to infer the hidden platform's location (via allocentric extrapolation) emerges. We also demonstrate that behavioural changes, towards a more allocentric strategy, are reflected in increases in hippocampal brain-derived neurotrophic factor. (C) 2007 Elsevier Inc. All rights reserved.
\end{abstract}

Keywords: Water maze; Spatial navigation; Allocentric; Hippocampus; BDNF

\section{Introduction}

The Morris water maze (MWM) task, initially described over 25 years ago, is a simple yet effective paradigm used in the investigation of spatial navigation, learning and memory in laboratory animals (Morris, 1981, 1984; Morris, Garrud, Rawlins, \& O'Keefe, 1982). Within this circular pool of water animals are given several training trials to locate a hidden escape platform. It's simplicity and ease of use offers this task many advantages over other spatial tasks. The use of water allows for control over odour cues and serves as an excellent motivating factor for animals to find and remember the location of the hidden platform. Despite these advantages and the simple nature of the task,

\footnotetext{
* Corresponding author. Fax: +35317084767.

E-mail address: Sean.Commins@nuim.ie (S. Commins).
}

how animals navigate toward and retain information regarding the location of the escape platform, remains unclear. Multiple navigational strategies in orientation and retention of spatial information have been illustrated in both animals and humans (Aggleton, Vann, Oswald, \& Good, 2000; Pearce, Roberts, \& Good, 1998). These strategies are broadly separated into those termed egocentric (defining the relation of an object, goal or location relative to the subject) or allocentric (defining the relation of an object, goal or location relative to another location, where this object is independent of the subject). Such egocentric mechanisms can also include, for example, repeating a particular sequence of responses or motor movements to a target, or learning to directly approach a distinct cue in the environment (Brown, 1992; de Bruin, Moita, de Brabander, \& Jooster, 2001) with little information on the spatial relations between the cues used. However, previous research has strongly implicated the use of allocentric 
strategies in solving the MWM, particularly the use of visual distal cues in forming spatial relations (Cheng, 1986; McGauran, Harvey, Cunningham, Craig, \& Commins, 2004). It is thought that integrating the spatial relationship of several distal cues to one another and to the platform's location (including the direction and distance between them) allows for accurate localisation of the goal (Benhamou \& Poucet, 1998; Hamilton, Rosenfelt, \& Whishaw, 2004). Indeed, rodents have been shown to successfully navigate the water maze using several external distal cues (Allen, 2004; Martin, Walker, \& Skinner, 2003), with later retention of the task dependent on the association between the cue configuration and the location of the platform (Cohen \& Bussey, 2003; McGauran et al., 2004).

One temporal lobe structure known to play an important role in spatial learning and memory is the hippocampal formation (Aggleton, Keith, Rawlins, Hunt, \& Sahgal, 1992; Jarrard, 1993; Morris et al., 1982). Lesion studies have demonstrated that an animal's ability to learn and retain the location of the hidden platform in the water maze task is impaired, despite the platform's position being marked by the arrangement of distal landmarks (de Bruin et al., 2001; Morris et al., 1982; Pearce et al., 1998). Although animals can still swim in the pool itself following these ablations, the vicinity of their searching is less accurate (de Bruin et al., 2001; Whishaw \& Jarrard, 1996) displaying little preference for the goal area itself. It is generally accepted that these impairments reflect the importance of the hippocampal formation in establishing allocentric relations (Morris et al., 1982). For example, the hippocampus is known to respond to the spatial arrangement of environmental cues (Hetherington \& Shapiro, 1997), with rotation of distal cues leading to a similar rotation of hippocampal place fields (Muller \& Kubie, 1987). In addition, the hippocampus also seems to be responsible for correct goal recognition (Hollup, Kjelstrup, Hoff, Moser, \& Moser, 2001). These factors amongst others are critically important for the formation of allocentric representations during the MWM task.

The water maze task, despite its simplicity and popularity of use, is nonetheless a complex behavioural task. In the hidden platform version of the task, animals must use a range of different strategies, ranging from swimming away from the pool wall, to discovering the platform, to climbing on the platform and remaining there before learning the platform location relative to the surrounding cues (Nakazawa, McHugh, Wilson, \& Tonegawa, 2004). Despite the accumulation of evidence suggesting that the water maze relies on the formation of allocentric relations, which in turn requires a functioning hippocampus, the question remains of how animals form allocentric representations of their environment using distal cues and can these representations be separated out in terms of hippocampal functioning? In the typically adopted spacedtraining version of the task (D'Hooge \& De Deyn, 2001) learning requires multiple training trials over many days, as such it is extremely difficult to state exactly how and when these spatial relations are formed. Many authors simply suggest that spatial relations are formed as the animal sits on the platform between each trial (Keith \& Mc Vety, 1988; Sutherland \& Linggard, 1982), while others suggest (Chapillon, 1999; Devan, Blank, \& Petri, 1992; Moghaddam \& Bures, 1996) that locomoting during the trial is crucial for relation formation. We would argue, however, that navigation in the water maze is dynamic and constantly changing and therefore solving the task may not rely solely on the formation of allocentric representations, but instead involves a combination of different strategies that include both cue-dependent allocentric and egocentric representations.

Here, we attempt to provide a comprehensive analysis of MWM acquisition, incorporating both the animal's swimming in the pool and time spent on the hidden platform, detailing a range of different behavioural strategies used that ultimately culminate in the formation of cue-dependent egocentric and allocentric representations. We further argue that the formation of different representations may change over time. As accurate learning of the platform's location requires many training trials, allocentric representations may not emerge until later in the training period (Cimadevilla, Wesierska, Fenton, \& Bures, 2001). If this is the case, then we would suggest that molecular changes in the hippocampal region reflecting alterations in the animal's behaviour during acquisition (particularly the formation of allocentric representations) may also appear later during the training period. To this end, we monitored one molecule known to be fundamentally involved in memory formation; brain-derived neurotrophic factor (BDNF, Lee, Everitt, \& Thomas, 2004; Tyler, Alonso, Bramham, \& Pozzo-Miller, 2002). As the highest expression of BDNF in the brain is found in the hippocampus, BDNF has also been strongly implicated in hippocampally dependent spatial learning (Hall, Thomas, \& Everitt, 2000; Yamada, Mizuno, \& Nabeshima, 2002). For example, Kesslak, So, Choi, Cotman, and Gomez-Pinilla (1998) demonstrated that water maze training resulted in increased expression of BDNF in the hippocampus, but not in the cerebellum, striatum or neocortex. Finally, BDNF's rapid expression during or soon after learning (Bekinschtein et al., 2007; Lee et al., 2004) via the Mitogen-Activated Protein-Kinase (MAP-K) signalling cascade pathway, in hippocampaldependent learning tasks (Tyler et al., 2002) makes this an ideal marker to examine changes that may reflect acquisition of the MWM overall.

\section{Experiment 1}

Our initial experiment aims to confirm that long-term retention of the water maze task depends on the retention of cue-platform associations. We hypothesise that by rotating the distal cues $180^{\circ} 7$ days post-acquisition will result in a similar rotation of searching by animals in the retention probe task. 


\subsection{Methods}

\subsubsection{Animals}

Male Wistar rats (250-350 g, Biomedical Unit, University College Dublin), 3 months old, served as subjects. Animals were in similar housing conditions to those previously reported (McGauran et al., 2004). Guideline for the maintenance and experimentation of animals conformed to the Department of Health and Children (Ireland) guidelines and European directive 86/609/EC.

\subsubsection{Apparatus and procedure}

Rats $(n=15)$ were trained ( 4 trials/day over 5 days) in a $170 \mathrm{~cm}$ diameter water maze $\left(20 \pm 1^{\circ} \mathrm{C}\right.$, maximum duration $60 \mathrm{~s}, 15 \mathrm{~s}$ platform interval, $15 \mathrm{~s}$ inter-trial interval (ITI)) to locate the platform (centre NE quadrant, $15 \mathrm{~cm}$ diameter, $1 \mathrm{~cm}$ below water level). Animals were transported from holding cage and placed into the water facing the pool wall, from one of four pseudorandom starting positions (north, south, east or west). Black curtains surrounded the pool $(50 \mathrm{~cm}$ from edge) with three distal cues in fixed positions outside the pool ( $60 \mathrm{~W}$ light bulb NE corner, $40 \mathrm{~W}$ light bulb NW corner and a white rectangular card $(55 \mathrm{~cm} \times 81 \mathrm{~cm})$ east side of pool) throughout experimentation. All acquisition trials were recorded using EthoVision (Noldus Information Technology, Netherlands).

Retention was assessed 7 days post-acquisition (see McGauran et al., 2004, for details). Rats were randomly assigned to one of two groups; controls (CT, $n=8)$ or cue-rotated group (CR, $n=8$ ). Retention for both groups was assessed by removing the platform and allowed to swim for $60 \mathrm{~s}$. For control group each rat was placed into the water maze at NW position. The distal-cue layout remained the same as during the acquisition phase. For cue-rotated group, each rat was again placed into the water at the NW position but the distal cues were rotated by $180^{\circ}$, so that one light was now suspended from the SW corner, and the other from the SE position. The sheet of white paper was now positioned on the western side. Measures of retention included mean percentage time (of the $60 \mathrm{~s})$ spent swimming by both groups in each of the four quadrants of the pool. Mean percentage time spent swimming in the 'platform area' was also assessed. This is an area of approximately $27 \mathrm{~cm}$ in diameter and centred at where the platform was located during the acquisition (middle of NE quadrant). Three other equivalent areas were also examined.

\subsubsection{Statistics}

Several repeated-measures ANOVAs with appropriate post-hoc tests (Tukey) were conducted, wherever required independent $t$-tests were calculated.

\subsection{Results}

All animals $(n=16)$ acquired the water maze task, with mean escape latencies reducing from
$45.78 \pm 2.53 \mathrm{~s}$ on the first training day to $19.96 \pm 2.85 \mathrm{~s}$ on the final day (Fig. 1a). A repeatedmeasures ANOVA confirmed this decrease to be significant across acquisition overall $(F(4,12)=13.599$, $p<.001)$. Retention of the water maze was assessed 7 days post-acquisition with a single probe trial. The control group spent significantly more time in the NE quadrant (i.e. the position of the platform during acquisition training) compared to the SW quadrant $(t(14)=2.11, p=.05)$ but importantly Fig. 1b demonstrates that the CT group spent significantly more time exploring the NE quadrant in comparison to the $\mathrm{CR}$ group $(t(14)=4.736, p<.001 ; \mathrm{CT}, 24.79 \pm 1.27 \%$; CR, $12.29 \pm 2.31 \%$ ). However, when the SW quadrant was examined, we found that the CR group spent significantly more time in this region when compared to the CT group $(t(14)=-4.415, p<.001 ; \mathrm{CT}, 17.79 \pm 3.06 \%$; CR, $37.7 \pm 3.3 \%$ ). Similar results were obtain for the 'platform area' with the CT group spending significantly more time swimming in the NE 'platform area' in comparison to the $\mathrm{CR}$ group $(t(14)=2.107, p<.05$; CT, $3.75 \pm 1.06 \%$; CR, $1.47 \pm 0.48 \%$ ), while the $\mathrm{CR}$ group spent significantly more time in the SW 'platform area' compared to the CT group $(t(14)=-2.141, p<.05$; CT, $2.13 \pm 1.44 \%$; CR, $7.29 \pm 1.93 \%$ data not shown).

\section{Experiment 2}

Results from experiment 1 confirm our previous findings (McGauran et al., 2004) that animals in the MWM establish a long-lasting association between the distal visual cues and the hidden platform's location. The results demonstrate that rotation of the cues $180^{\circ} 7$ days postacquisition leads to a concomitant rotation of searching during the retention probe trial. This finding led us to ask how are such cue-platform associations formed? Research has indicated that this association may be acquired either when animals sit on the platform (Keith \& Mc Vety, 1988) or during locomotion in the trial (Chapillon, 1999; Devan et al., 1992; Moghaddam \& Bures, 1996). To examine both scenarios, in a second experiment, we trained animals to find the platform and analysed their behaviour while they sat on the platform during the inter-trial interval and also during locomotion in each trial.

\subsection{Methods}

\subsubsection{Animals}

Male Wistar rats (250-350 g, Biomedical Unit, University College Dublin), 3 months old were again used in this experiment and were housed in conditions similar to those reported in experiment 1.

\subsubsection{Apparatus and procedure}

All animals $(n=8)$ were trained for 5 days (4 trials/day) in the MWM task as reported in experiment 1. 
a
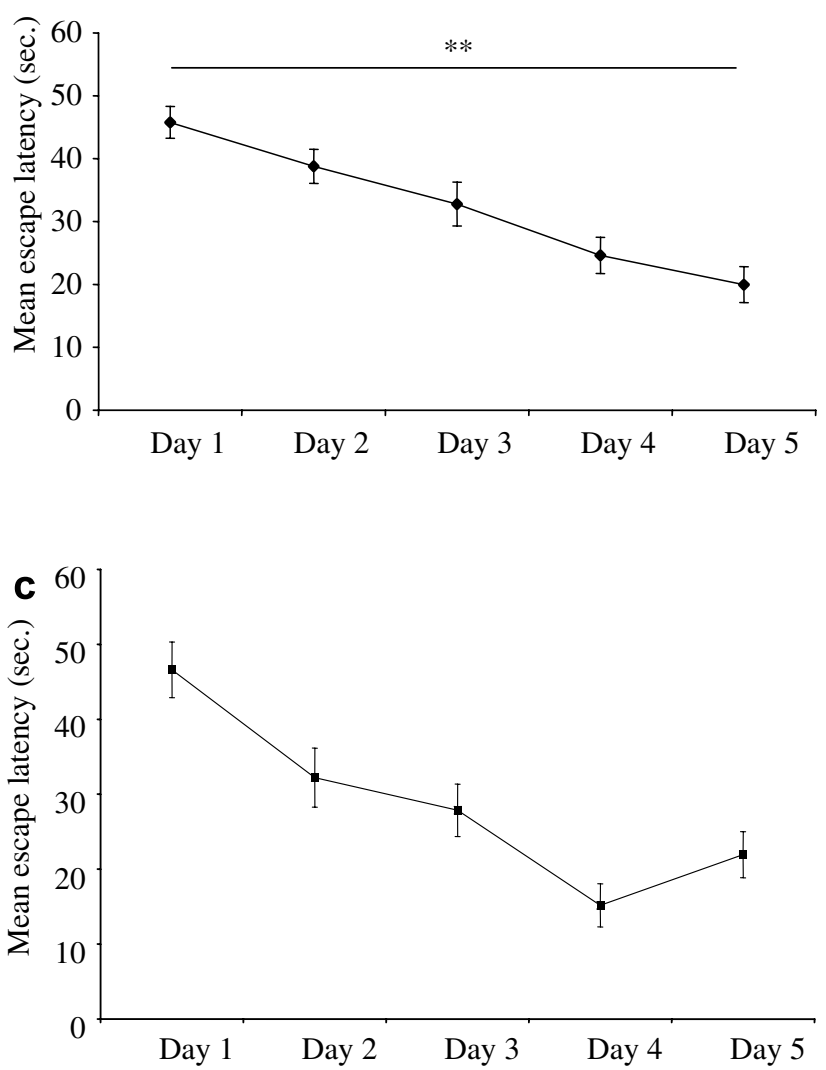

b

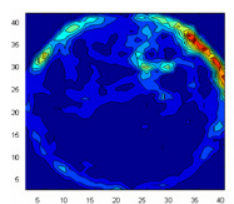

Controls

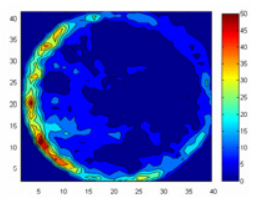

Cue-Rotated

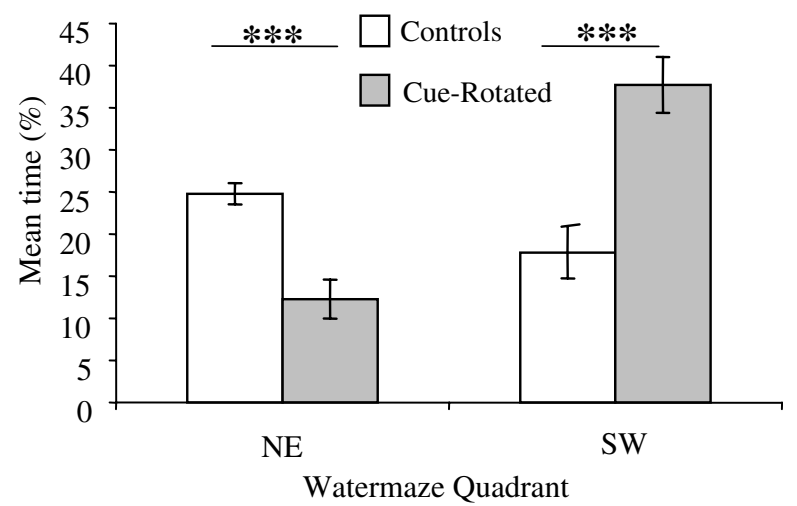

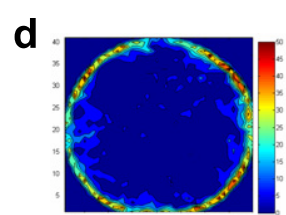

Day 1

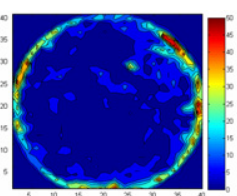

Day 2

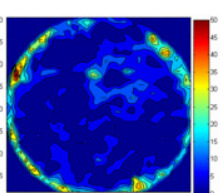

Day 3

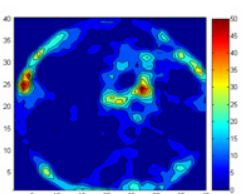

Day 4

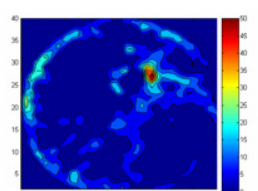

Day 5

Fig. 1. (a) Mean escape latency ( $\pm S E M$ ) for animals having been trained to search for the platform in NE quadrant and (b) Mean percentage time $( \pm S E M)$ spent in NE and SW quadrants for the control and cue-rotated groups ( $n=8 /$ group) during retention probe trial 7 days post-acquisition ${ }^{* * *} p<.001$, with Matlab density plots of overall search activity during retention (colour-coded red to blue representing high to low search activity respectively). (c) Mean escape latency $( \pm S E M)$ with (d) Matlab density plots during acquisition $\left({ }^{*} p<.05,{ }^{* *} p<.01,{ }^{* * *} p<.001\right)$.

\subsubsection{Head-direction analysis}

All platform intervals $(15 \mathrm{~s})$ were digitally recorded using an overhead camera with video capture software (VirtuaDub 1.5 10), and segmented (TMPGEnc 2.5, Hiroyuki Hori/Pegasys Inc.) to produce 15 digital photographs (1 s apart) for each trial for each animal ( $n=2400$ total). The head direction was calculated using Adobe Photoshop 5.0, based on a line running from the midpoint between the animal's eyes to the tip of the snout. Using the most southern point on the platform as $0^{\circ}$, all head directions of each animal were plotted and analysed.

\subsubsection{Data analysis}

3.1.4.1. Correlation of platform head direction to subsequent swim trial. The water maze was sectioned into 36 segments $\left(10^{\circ}\right.$ increment apart, platform as centre), with the first segment extended vertically from $0^{\circ}$ on the platform $\pm 5^{\circ}$ to the pool edge. All head-direction data were re-entered in the corresponding segment and calculated as an overall percentage of time spent orientated in that segment. EthoVision provided the percentage of time spent in each segment during swimming. Due to unequal surface area in each segment, theoretically animals would be more likely to be found in larger segments due to increased size, therefore the percentages given by EthoVision would be biased accordingly. To counteract for this, segment percentages were normalised based on their respective surface area. Oriana (version 2) calculated the mean segment that was occupied for both data sets in each trial, providing the data used for correlations. Mean heading direction (calculated by EthoVision) was defined as the initial definite directional movement made within the first $15 \mathrm{~s}$, beginning at the respective starting location. Disorientation and adjustment to the water environment was accommodated by eliminating the first $5 \mathrm{~s}$ of the trial due to the short stochastic nature of movements presented during this time. Successful trials under $5 \mathrm{~s}$ all had identifiable initial heading directions therefore were used. 
3.1.4.2. Measurement of angular cue configuration on animal's head during trial. EthoVision provided $x, y$ coordinates $(0.2 \mathrm{~s}$ increments apart) for the animals position throughout each trial which were drawn in a scatterplot with the coordinates for each cue. The cue angle was calculated $(0.2 \mathrm{~s}$ increments apart) throughout the trial using basic trigonometric formulae. The cue angles were subsequently graphed. Simultaneously evaluating the position of each cue on each graph and confirming it via observation of the recorded track in EthoVision assessed the momentary behavioural strategy used.

3.1.4.3. Zones of the pool. To examine turn positions, the pool was divided into three zones of equal area, based on the coordinates of each turn. For each turn point, a line was drawn between the centre of the platform, the turn position and the pool edge using Adobe Photoshop 5.0. This program also measured the length of each line. The location of the turn on each respective line was normalised giving a percentage position on that line as to where the point lay (i.e. pool wall was at $100 \%$ and platform edge was at $0 \%$ ). This accounted for unequal distances from the platform to the side. Three zones were used in order to categorise the location of turn positions in the pool; turns within $0-33 \%$ were in the "near" zone. Turns within $34-66 \%$ were in the "middle" zone. Turns within $67-100 \%$ were in the "far" zone.

\subsubsection{Statistics}

Several repeated-measures ANOVAs with appropriate post-hoc tests (Tukey) were conducted, wherever required independent $t$-tests were calculated. When reporting correlations, Pearson's correlation coefficient $(r)$ was used. Circular statistics were used to assess all collated raw data of a non-linear nature (e.g. head direction, mean segment searched) with the appropriate Rayleigh tests and Watson-Williams $F$-tests.

\subsection{Results}

\subsubsection{Characterisation of head-direction movements on} platform and prediction of subsequent searching behaviour

Fig. 1c and demonstrates that all animals $(n=8)$ in this experiment successfully acquired the task $(F(4,35)=8.095, p<.001)$. As each animal acquired the task we recorded the head directions of each as they sat on the platform for $15 \mathrm{~s}$ between trials (giving $2400 \mathrm{sepa}-$ rate $1 \mathrm{~s}$ head-direction stills, see Section 3.1). Fig. 2a and $\mathrm{b}$ demonstrates that most platform head movements occur after the first trial but by trial 4 they decrease or stop. There is a significant decrease in head movements from trial 1 to trial 4 on Days 1, 2, 4 and 5, respectively (Day 1: $\quad F(3,21)=12.842, \quad p<.01 ; \quad$ Day $2: \quad F(3,21)=4.81$, $p<.01 ; \quad$ Day 4: $F(3,21)=3.84, \quad p<.05 ; \quad$ Day 5: $F(3,21)=4.959, p<.01$; there was no significant decrease on Day 3: $F(3,21)=0.778, p>.05)$. In addition, there was no significant change in the mean daily range across all 5 days $(F(4,35)=1.732, p>.05)$ suggesting a similar behaviour across all days. Fig. $2 \mathrm{c}$ and d also illustrates animals generally tended to look around the arena over the 5 days, only showing a significant preference in orientation towards the cues on 2 days (Days 3 and 5; both Rayleigh, $p<.01)$. In an attempt to examine whether a larger viewed range (of head-direction movement) would predict successful navigation, each animal's range of head movement on the platform was correlated with their escape latency on the subsequent trial, but found no effect $(r=-.014$, $p>.05$ ). Furthermore, we also did not find a significant relationship between the amount of time spent looking at a cue and performance on the subsequent trial $(r=-.109, p>.05)$.

We did however find that the direction in which an animal looked while sitting on the platform predicted searching behaviour on the next trial. On Day 1, for example, an animal's mean head direction on the platform significantly correlated with the mean location searched at in their subsequent trial $(r=.603, p=.002$, see Section 3.1). This effect was also time-dependent with the first $20 \mathrm{~s}$ being most strongly correlated (Fig. 2e). The majority of animals on Day 1 did not find the platform, with their swimming and searching confined to the pool edge. However, animals on some trials $(10 / 32)$ were successful, and their searching behaviour could also be attributed to the direction orientated towards during the platform interval. The mean head direction of an animal on the platform correlated significantly $(r=.805, p<.01)$ with their initial mean heading direction during the subsequent successful trial highlighting their early progression away from the pool wall during these occurrences. On Days 2, 3, 4 and 5 animals were more successful at finding the platform and showed less propensity to search/stay at the pool edge (mean percentage time at side decreased from $75.88 \%$ on Day 1 to $41.50 \%$ on Day $5 ; F(4,35)=6.338, p<.001)$. Although still significant, the correlation between the mean head direction presented on the platform and the initial heading direction in the proceeding successful trial decreases from Days 2 to 5 (Fig. 2f), suggesting that information proffered during the platform period dwindles.

\subsubsection{Characterisation of cue-dependent searching strategies used during trial locomotion}

In addition to recording the platform head-direction movement we also recorded each animal's movement during each trial for the 5 training days. To elucidate how distal visual cues are used during a trial, we calculated, the angle each of the three cues subtended with the animal's head during movement in each successful trial $(n=10$, 23, 26, 29 and 30/32 trials for Days 1-5, respectively, see Section 3.1 and Judd \& Collett, 1998). From this resulting information, five distinct cue-related searching strategies emerged; cue stability, turning, scanning, approach and movement away from cues. The first behaviour, referred to as "stability", is characterised by animal movements in a definite direction over a minimum period of $1 \mathrm{~s}$ (five 


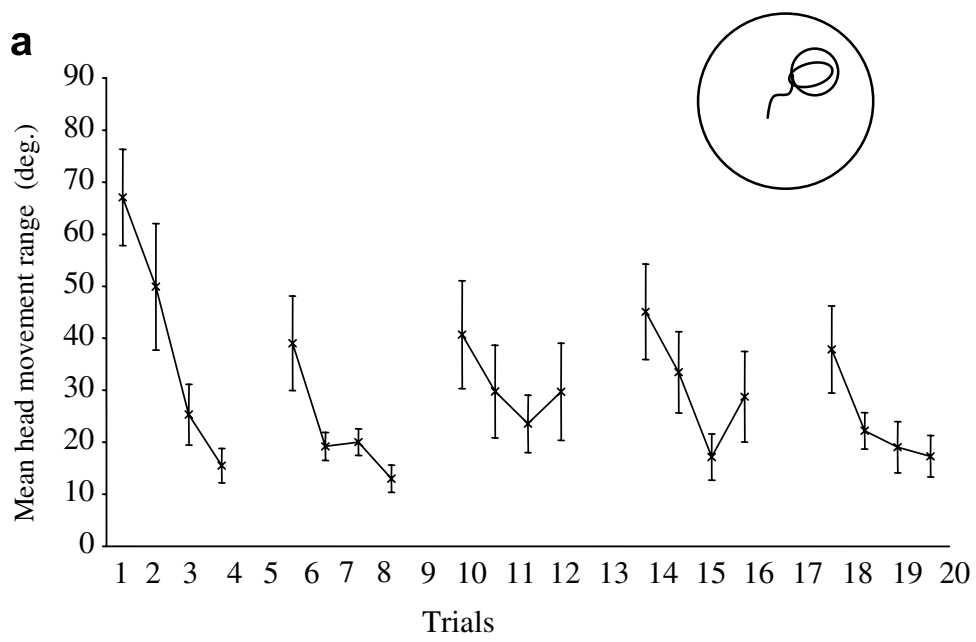

b

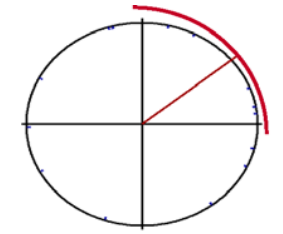

Trial 1

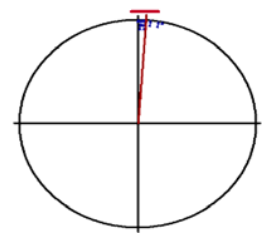

Trial 4
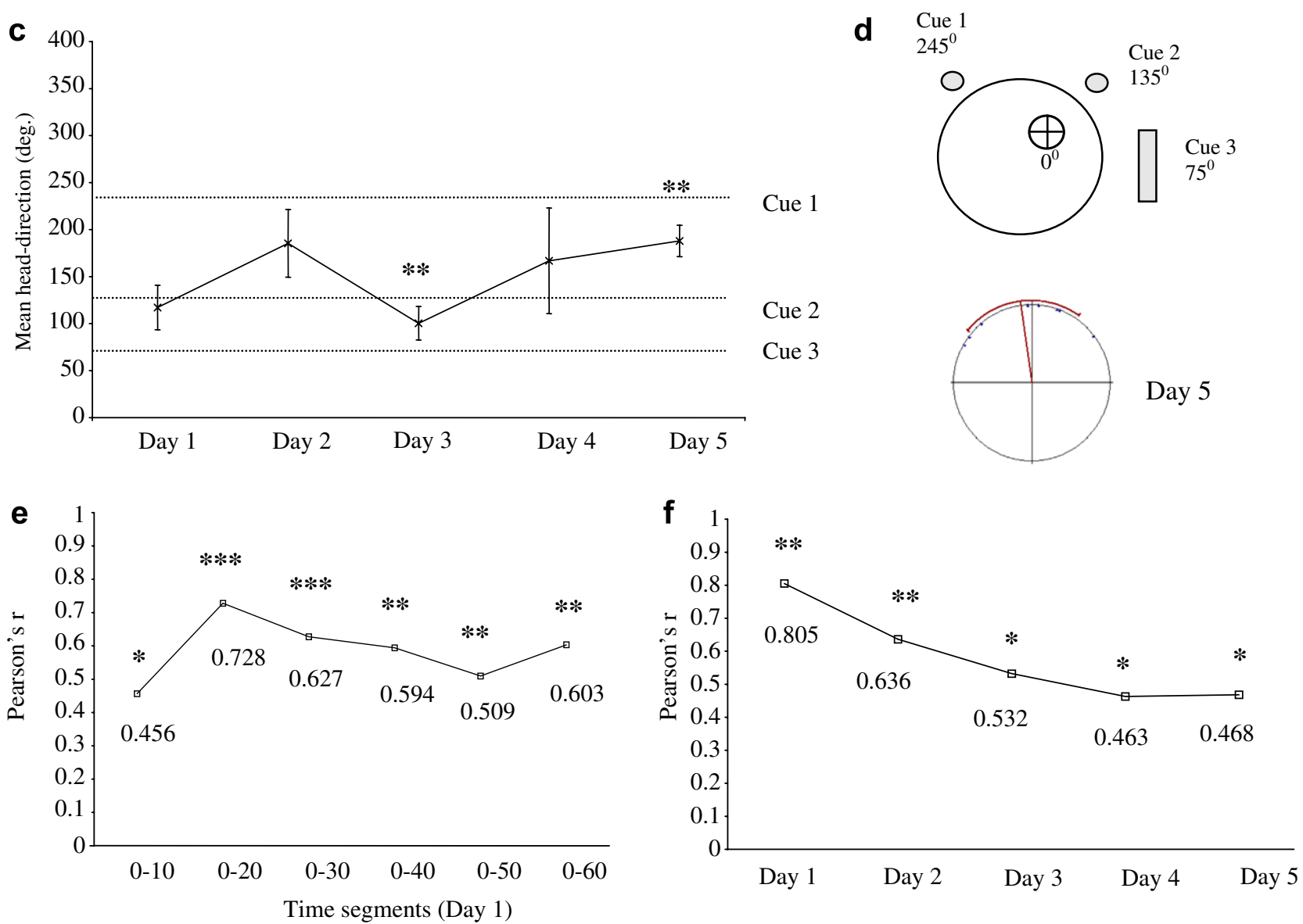

Fig. 2. (a) Mean range of head movements on the platform ( $\pm S E M$ ) (inset: schematic of the animal's position on the platform). (b) Circular plots of trials 1 and 4 as examples of the decrease in mean range of head movements. (c) Mean head direction $( \pm S E M)$ on the platform (Rayleigh Uniformity test, $\left.{ }^{* *} p<.01\right)$. (d) Circular plot of the overall mean head direction on Day 5 (inset: schematic diagram of the cue positions relative to the platform). (e) Pearson's correlation of the mean orientation of an animal on the platform to mean location searched during the subsequent trial broken into time intervals for Day 1. (f) Correlation of the mean orientation of an animal on the platform to the initial mean heading direction on the subsequent trial across acquisition.

consecutive data points, each data point is $0.2 \mathrm{~s}$ ), where the cue(s) position is maintained at a constant angle $\left(< \pm 20^{\circ}\right)$ to the animals head, while moving towards a cue. (This "stability" behaviour is comparable to the "pivoting" action reported in the wood ant (Formica rufa) when approaching a landmark (Judd \& Collett, 1998)). The second behaviour (i.e. "approach") is observed when the position of the cue in relation to the animal's head becomes increasingly/ decreasingly centralised $\left(< \pm 20^{\circ}\right)$ at a constant rate, through movements in a specified direction towards a 
cue. Although both stability and approach behaviours are outlined independently, they often are detected together and can be treated as one (see Fig. 3a, first panel) due to both moving positively in the direction of a cue. The third behaviour is typified by a turn. For this behaviour, the animal moves in one distinct direction, with a cue centralised and/or stable, followed by a change in orientation signified by movement in a second distinct direction, with a second cue becoming stable and/or centralised in relation to the animals head (Fig. 3a, second panel). The fourth distinguishable behaviour sees the animal moving away from the visual cues, thereby increasingly/decreasingly decentralising the cue(s) position. The final behaviour, referred to as "scanning", is illustrated by animal movements in a more general direction, while maintaining a centralised cue(s) angle from the animal's head, yet the cue position is not "stable" but rather meanders up and down in a constant manner $\left(< \pm 50^{\circ}\right)$ around this core angle, during a minimum period of $1 \mathrm{~s}$ (Fig. 3a, third panel). A similar scanning behaviour has been previously outlined in bees and Cerceris wasps upon leaving and returning to a goal (Zeil, Kelber, \& Voss, 1996). a
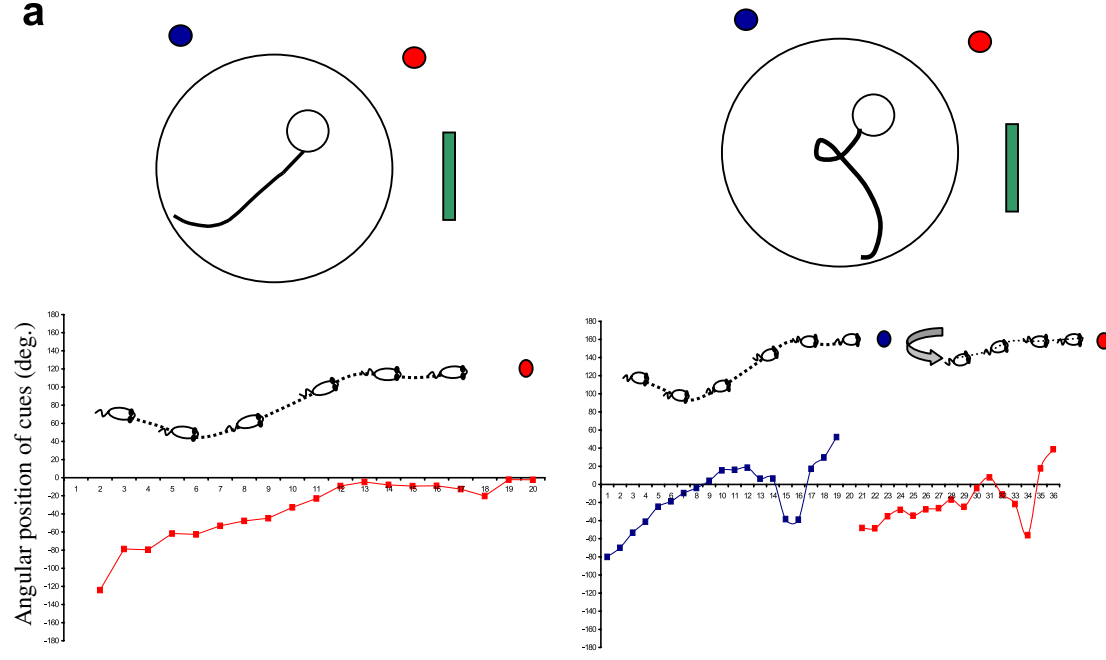
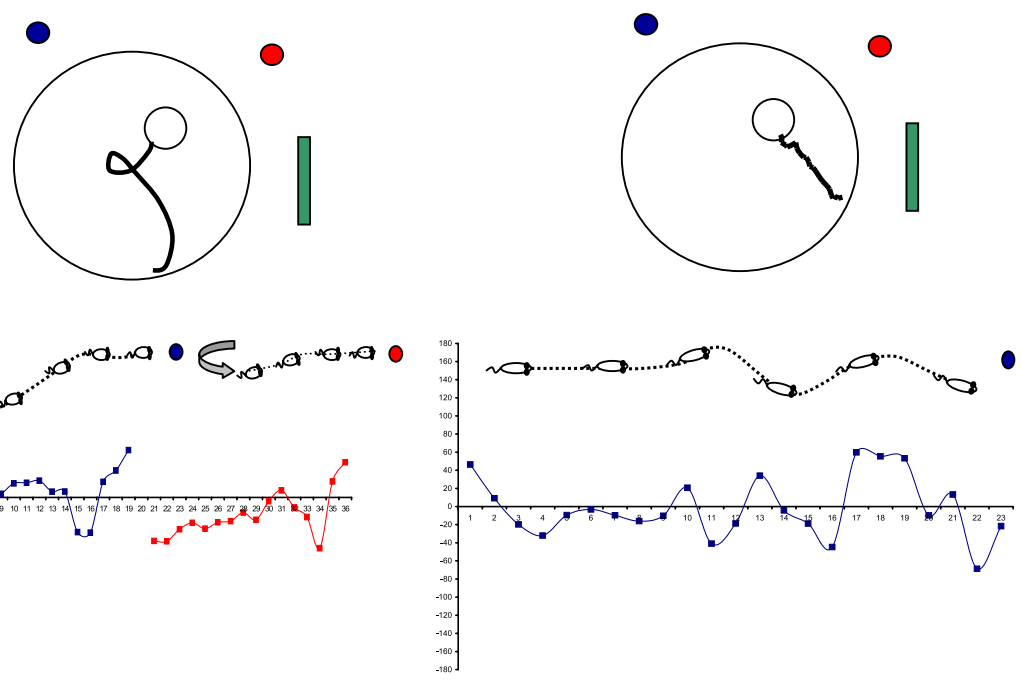
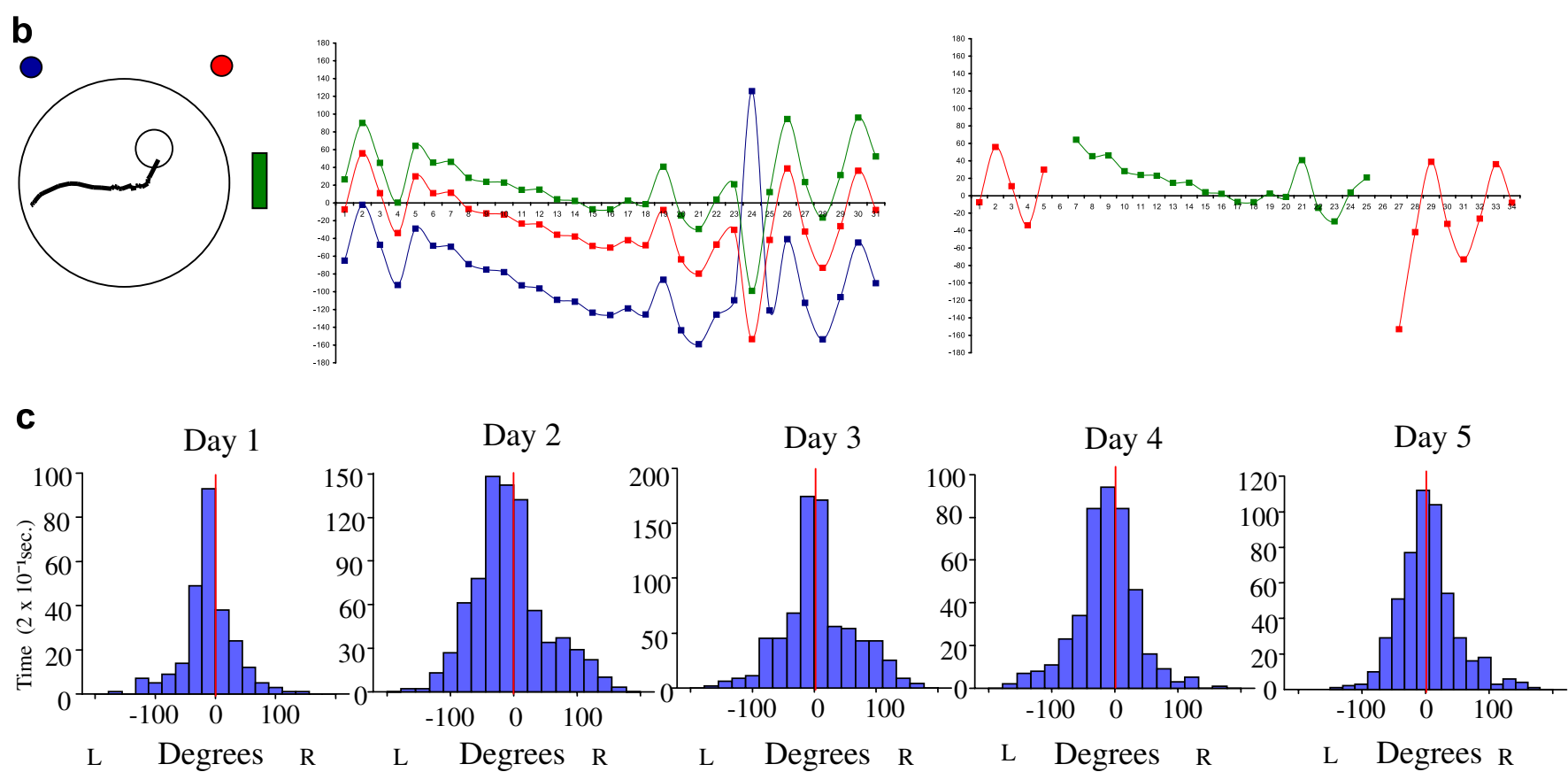

Fig. 3. (a) The position of cues relative to the animal's head during three successful trials, demonstrating the momentary behavioural strategies used throughout. Schematic diagrams (inset) illustrate the animal's position in the water maze relative to the cues during each respective trial. (first panel) Initial approach behaviour leading into stability behaviour towards the red cue. (second panel) A turning episode between a series of approach and stability behaviours in the direction of the blue and red cues, respectively. (third panel) Scanning behaviour in the direction of the blue cue (see insets above for schematics of the animals swim). (b) A successful trial, demonstrating the animal's momentary behavioural strategies (cues 1, 2 and 3 colour-coded blue, red and green, respectively). Animal swim track (first panel), with the angular position of all cues relative to the head during this successful trial (second panel). The most central cue in the visual field $\left(\sim 0^{\circ}\right.$, minimum period $\left.1 \mathrm{~s}\right)$ used momentarily throughout the trial (third panel). (c) Histograms showing the position of all cues relative to the animals head during approach and stability behaviour, across days. 


\subsubsection{Using the cue-based strategies egocentrically and allocentrically to find the hidden platform}

Although no pattern of strategies is replicated within or between animals, by employing these behaviours independently or in combination an animal can effectively locate the hidden platform. Fig. 3b outlines an example of a successful trial. Fig. $3 b$ (first panel) shows the actual track of the animal in the maze, with Fig. 3b (second panel) demonstrating the changes in angular position that each of the cues make with the animals head during the successful trial. Taking the cue most central in the animal's visual field $\left(\sim 0^{\circ}\right)$, at any time we can break any trial into one or more of the above-mentioned strategies (Fig. 3b, third panel). In the example provided, the animal initially scans towards cue 1 (red), followed by a turn and movement in the direction of cue 3 (green), before turning and scanning again towards cue 1 (red). From this analysis, animals seem to head directly towards the cues, either turning towards a cue and keeping it stable on the retina for a while, or they move towards a cue in a scanning motion. If animals tend generally to move towards the cues, by measuring the angular position of each cue on the animal's head during approach and stability we should expect a frequency distribution of angles concentrated around zero for all successful trials. Fig. $3 \mathrm{c}$ confirms this to be so, with similar results found for each individual cue across days (data not shown).

Heading directly towards the cues using an "egocentricguiding" strategy is useful but might not always guarantee a successful outcome because the platform is located at a distal point from the cues. This strategy is viable when animals move along a route that links both platform and cue. The animal must gain some complementary information in order to discern the exact location of the platform in relation to the overall configuration of cues, as opposed to a wider general vicinity provided by cue approach alone. "Scanning" behaviour could potentially provide this supplementary information, while still approaching a cue. It may also give the animal more familiarity with the environment (Zeil et al., 1996) as well as an increased probability in finding the hidden target, due to the wider surface area and views covered compared to "stability" searching. This "scanning" behaviour initially emerged on Day 2, by which time the animals would have awareness of the platforms existence, in addition to a rudimentary knowledge of the cue layout from the previous encountered trials and platform intervals. Only four episodes of scanning were recorded initially but this figure increased, with 6 noted on Day 3, 12 on Day 4 and 8 on Day 5 (see Fig. 4a for a spatial distribution of scanning incidents). Furthermore, the mean arc viewed on each scanning episode increased over training $(F(3,26)=3.129, p<.05$, Fig. $4 b)$. However, scanning behaviour is not witnessed consistently in all animals for each day and the mean number of scanning occurrences remained low and relatively unchanged across the acquisition period $(0.125 \pm 0.047, \quad 0.218 \pm 0.08$, $0.375 \pm 0.16$ and $0.25 \pm 0.09$ for Days 2, 3, 4 and 5, respec- tively, ANOVA: $F(3,28)=0.935, p>.05)$. Consequently scanning does not appear to be the main strategy employed to get to the platform with most efficiency.

The behaviour that readily demonstrates animals can infer the platform's position relative to the cues (allocentrically) is the turning strategy. Here, an animal heads towards a cue but readjusts its position and approaches another until the platform is found. Turning, unlike the other observed behaviours, was identified in all animals throughout training (Each animal makes 5 or more turns daily on Days 2, 3, 4 and 5. It is only on Day 1 of training that 2 of the animals do not show evidence of turning (Rats 7 and 8)). Fig. 4c plots the spatial distribution of each of these turns for each training day. With training, more turns are made overall $(F(4,14)=9.459, p<.001)$, with turns generally located at the pool edge ('Far-zone' see Section $3.1 ; F(2,14)=23.331, p<.001$; Fig. $4 \mathrm{c}$ and d). However, Fig. $4 \mathrm{~d}$ also demonstrates that with training the mean number of edge turns decreases (from Day 2 to 5) while simultaneously there are increases in middle and/or near zones turns. The mean number of 'far' turns was significantly higher than either 'middle' $(t(7)=-3.99, p<.05)$ or 'near' turns $(t(7)=-3.685, p<.05)$ on Day 2 , with a similar effect for Day 3 (Middle; $t(7)=-5.005, p<.01$; Near; $t(7)=-6.523, p<.01)$. This effect is gone for Days 4 and 5 (Fig. 4d), suggesting that, with training, animals find the platform effectively due to rising reorientation attempts closer to the platform. In addition, the turns for each cue are concentrated in precise regions of the pool (Fig. 4c and e). Distributions of turns towards cue 1 (blue) concentrate consistently SE of the platform (Rayleigh $p<.01$ for Days 1-5, Fig. 4e), with no significant difference in mean location on Day $1\left(47.4 \pm 23.25^{\circ}\right)$ compared to Day 5 $\left(48.73 \pm 14.06^{\circ}\right.$, Watson-Williams $F$-test, $F(1,43)=0.003$, $p>.05$ ). Distributions of turns towards cue 2 (red) also remained constant over training (mainly $\mathrm{S}$ of the platform; Rayleigh $p<.001$, for each day, Fig. 4e), with no significant difference between the mean turning distribution on Day $1\left(310.21 \pm 13.96^{\circ}\right) \quad$ compared to Day 5 $\left(336.66 \pm 7.76^{\circ} ; F(1,36)=3.25, p>.05\right)$. Turns for cue 3 (green) also demonstrated a preferred direction (mainly NW; Rayleigh $p<.001$ for each day, Fig. 4e), with a significant change in the mean location of these turns from Day $1 \quad\left(214.51 \pm 17.69^{\circ}\right)$ to Day $5 \quad\left(316.01 \pm 14.6^{\circ}\right.$; $F(1,60)=15.64, p<.001)$. This change may reflect the larger surface area occupied by this cue (large white cardboard sheet, compared to the two lights of cues 1 and 2) and the contrast of its edges with the black background. With training, the observed increases in turns particularly near the platform coupled to their consistent distribution allow animals to find the platform by combining egocentric guidance with allocentric inferences.

\section{Experiment 3}

Our findings thus far demonstrate the requirement of distal visual cues in overall acquisition of the MWM task. 
a
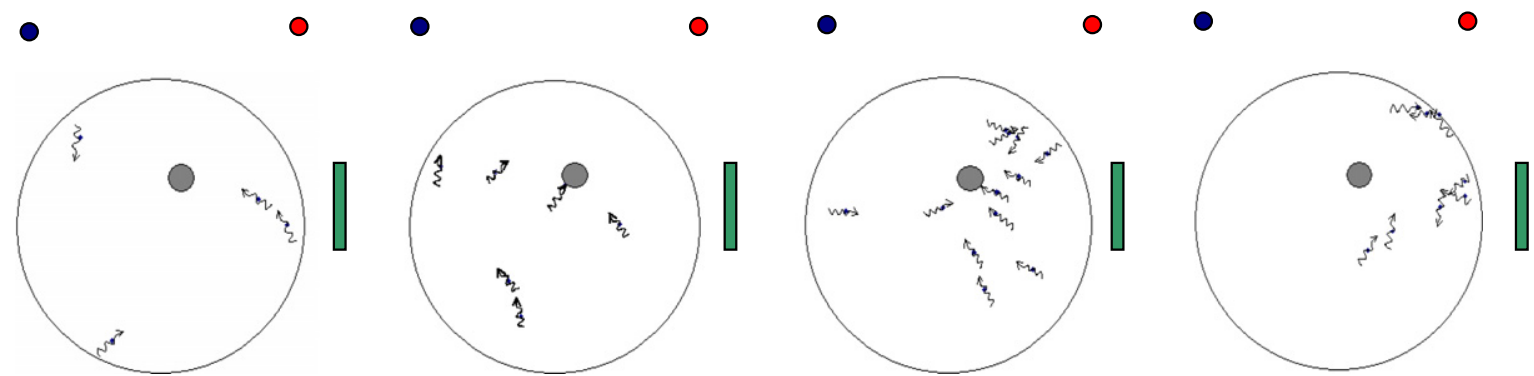

Day 2

Day 3

Day 4

Day 5

b

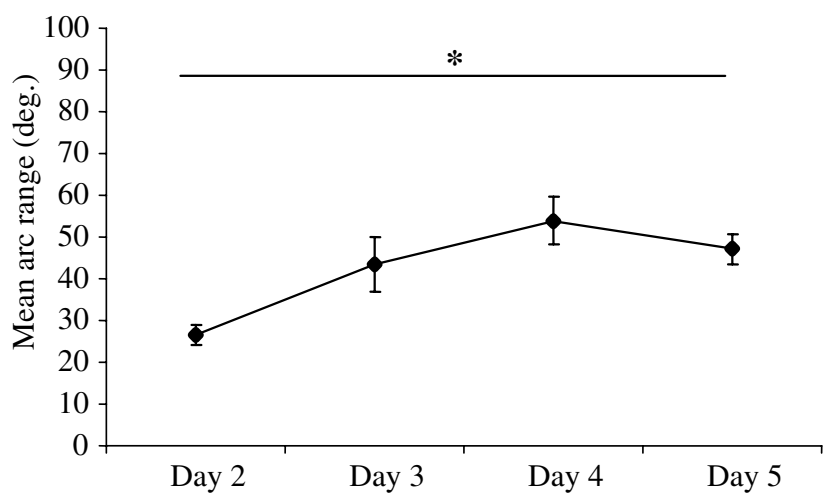

C

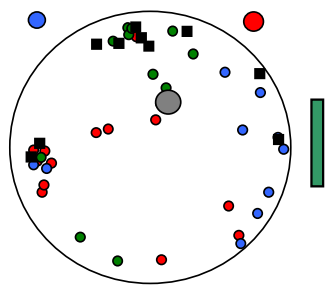

Day 1

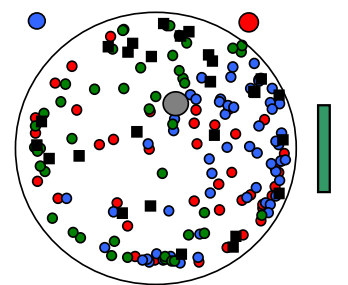

Day 2

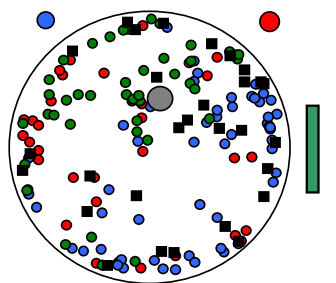

Day 3

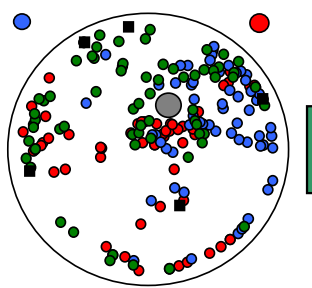

Day 4

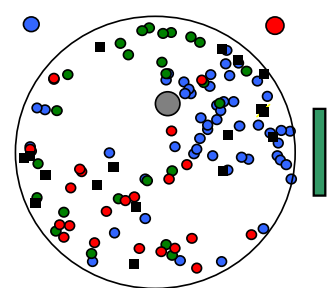

Day 5
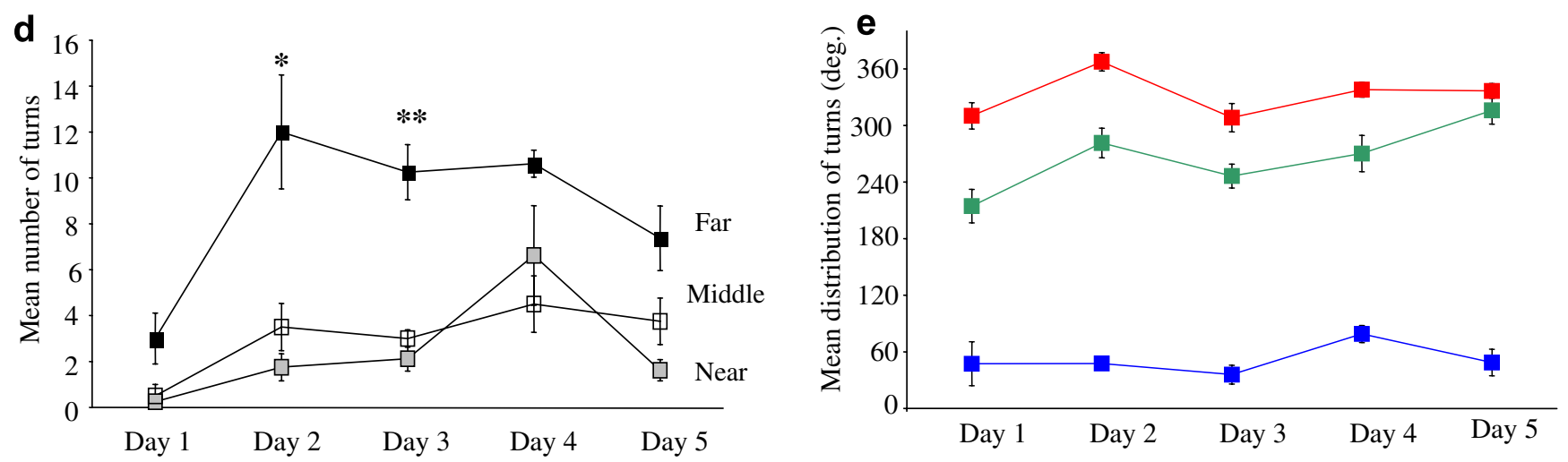

Fig. 4. (a) Scatterplot of spatial location of scanning in the water maze. (b) Mean range of scanning arc across acquisition ( $\pm S E M$ ). (c) Scatterplot of spatial location of cue turns (colour-coded, to identify the cue approached) and away turns (black) for each day. (d) Mean number of turns ( $\pm S E M$ ) made in zones of the pool (see Section 3.1). (e) Mean location of turns ( $\pm S E M$ ) relative to the platform (South $=0^{\circ}$ ), for all cues (i.e. blue, red and green).

Additionally animals incorporate these cues into their overall searching using several identified swimming strategies. At the same time, assessments of platform behaviour inbetween trials also indicate that animals gain valuable information during this interval. In experiment 3 , we inves- tigate the relative contribution the locomotion phase and platform interval offer to overall establishment of cue relations. Specifically, we ask whether exposure to distal visual cues during locomotion is sufficient for MWM acquisition. 


\subsection{Methods}

\subsubsection{Animals}

Male Wistar rats $(n=60$; Biomedical Unit, University College Dublin), approximately 3 months of age, weighing 250-300 g, at start of experimentation, served as subjects in this study and were housed in conditions similar to those reported in experiment 1 .

\subsubsection{Apparatus}

The hidden platform version of the MWM task was again used in this experiment. The three distal visual cues and submerged escape platform (centre of the NE quadrant) remained fixed in the same position throughout experimentation. A red LED light, powered by an attached water resistant battery, was used to track the movements of subjects in the water maze during darken conditions (Commins, Gemmell, Anderson, Gigg, \& O’Mara, 1999; Liu, Turner, \& Bures, 1994; Moghaddam \& Bures, 1996). For this, sticky backed Velcro was attached to the fur of the animals back, just below the neck. A thin elastic band was then placed over the subject's head and front paws and positioned over the Velcro. The reverse piece of Velcro was attached to the battery. The LED light, with the attached battery was then securely mounted onto the Velcro on the subjects back.

\subsubsection{Procedure}

All animals $(n=60)$ received four trials per day, for four consecutive days in the MWM task. These were divided into four groups. The first group (ON/ON, $n=15)$ had lights on during the locomotion phase in the pool, as well as when on the hidden escape platform (i.e. platform interval). The locomotion phase commenced when the animal was placed into the pool and finished when the platform was located. The platform interval started once the animal located the actual platform and was completed $15 \mathrm{~s}$ later. Therefore, the ON/ON group had access to the distal visual cues, throughout the entire acquisition trial. The second group (ON/OFF group, $n=15)$, had lights on during the locomotion phase only. Once the hidden escape platform was found all lights were turned off. The third group, (OFF/ON, $n=15)$, had no lights on during the locomotion phase. However, once they reached the hidden escape platform, the experimenter turned on the lights. Group 4 (OFF/OFF, $n=15)$, completed the entire acquisition trial in total darkness.

As the lights were extinguished in the MWM during the locomotion phase for two of the experimental groups (OFF/ON and OFF/OFF groups), to monitor tracking a lightweight LED light was attached to their backs via an elastic band during acquisition (see Moghaddam \& Bures, 1996). The remaining ON/ON and ON/OFF groups had a similar elastic band in order to control for any potential differences this band might cause in the animals performance. All animal movements were again captured using the EthoVision tracking program providing daily escape latencies, total distances travelled, and mean velocities.

\subsubsection{Statistics}

Several repeated-measures ANOVAs with appropriate post-hoc tests (Tukey) were conducted. Where required independent $t$-tests were calculated. A star-based system representing $p$-values of $*<.05, * *<.01, * * *<.001$, respectively, was used throughout.

\subsection{Results}

\subsubsection{Exposure to distal visual cues during locomotion is sufficient for Morris water maze acquisition}

Fig. 5 demonstrates the overall escape latencies for each group across all days. A $4 \times 4$ mixed factorial ANOVA revealed an overall main effect for experimental day $(F(3,141)=16.87, p<.001)$ and main effect for group $(F(3,47)=27.67, \quad p<.001)$. Subsequent post-hoc tests (Tukey, $p<.05)$ showed the ON/ON group $(26.31 \pm$ $1.41 \mathrm{~s})$ was significantly faster than the $\mathrm{OFF} / \mathrm{ON}$ $(50.28 \pm 1.19 \mathrm{~s} ; p<.001)$ and OFF/OFF groups $(48.19 \pm$
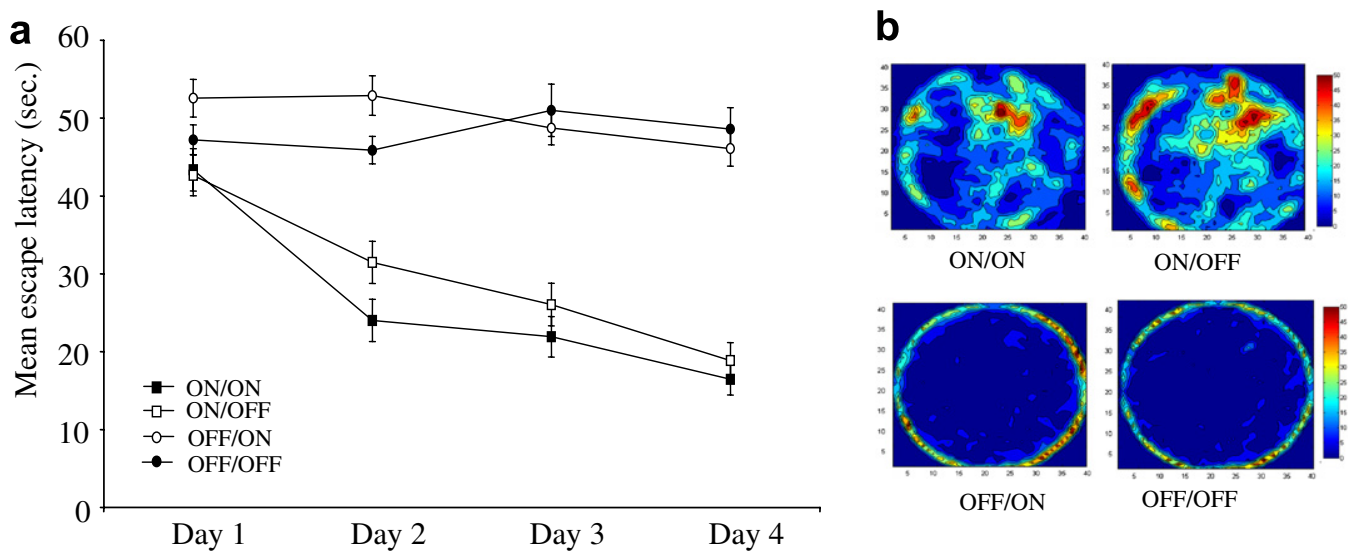

Fig. 5. (a) Mean escape latency ( $\pm S E M$ ) for the ON/ON, ON/OFF, OFF/ON and OFF/OFF groups with (b) Matlab density plots of search activity on Day 4. 
$1.17 \mathrm{~s} ; p<.001)$ overall. The ON/OFF group $(29.84 \pm$ $1.41 \mathrm{~s})$ was also faster than the $\mathrm{OFF} / \mathrm{ON}(p<.001)$ and OFF/OFF $(p<.001)$ groups overall. A main interaction effect was also found between the experimental day and group $(F(9,141)=5.664, p<.001)$.

These results suggest that although exposure to cues on the platform is important for directing navigation (particularly on the subsequent trial, see experiment 2), this period alone is insufficient to learn the platform's location. Conversely, exposure of cues during locomotion in a trial however is sufficient for animals to acquire the task and to form cue-platform associations.

\section{Experiment 4}

As stated in Section 1 one key brain structure implicated in spatial navigation, learning and memory is the hippocampal formation (Morris et al., 1982). To determine whether the behavioural changes that we observed as the animals learn cue-platform associations (experiment 2) are reflected in underlying molecular changes within the hippocampus we decided to monitor Brain-Derived Neurotrophic Factor (BDNF) expression in both the acquisition and retention of the MWM. This molecular marker was chosen because BDNF is well known to be involved in signalling during or soon after learning (Bekinschtein et al., 2007), it also has the highest expression (of the whole brain) in the hippocampal formation (Yamada et al., 2002) and has been implicated in contextual (Hall et al., 2000) and spatial memories (Mizuno, Yamada, Olariu, Nawa, \& Nabeshima, 2000).

\subsection{Methods}

\subsubsection{Animals}

Male Wistar rats approximately 3 months of age, weighing $250-300 \mathrm{~g}$, at start of experimentation, served as subjects in this study and were housed in conditions similar to those reported above.

\subsubsection{Apparatus and procedure}

All animals were trained in the MWM task as reported in experiment 1 . To examine changes in BDNF expression with acquisition five separate groups were used $(n=5 /$ group), Group 1 was trained for 1 day (4 trials), Group 2 for 2 days (4 trials/day), Groups 3, 4 and 5 for 3, 4 and 5 days, respectively. After each experiment, the animals were sacrificed, decapitated quickly and the brain was removed. The hippocampus and EC were dissected out according to standard methods (Gooney \& Lynch, 2001; Gooney, Shaw, Kelly, O'Mara, \& Lynch, 2002) and chopped and stored $\left(-70^{\circ}\right)$ pending subsequent biochemical analysis. Concentrations of BDNF were determined using ELISA. In order to disassociate BDNF involvement in physically swimming the MWM to it's involvement in learning the platform's location, we also allowed three groups of rats to swim (without a platform) for 1,3 or 5 days ( $n=4$ /group) for equivalent time-matched trials based on the mean for those trained with a platform for 1,3 or 5 days (see above). The hippocampus and EC were also dissected from each of these groups.

To examine changes in hippocampal BDNF expression with MWM retention five separate groups were again used ( $n=4$ /group). Group 1 was trained for 1 day (4 trials), given a 7 day rest followed by a retention probe task, similarly Groups 2, 3, 4 and 5 were trained for 2, 3, 4 and 5 days, respectively (4 trials/day), with each group also given a weeks rest followed by the retention probe task. Retention was assessed by removing the platform and allowing animals to swim for $60 \mathrm{~s}$. The mean percentage time in the 'platform area' was calculated (see Fig. 7a, inset and experiment 1). Following the retention test for animals that had received 1, 3 and 5 days training the hippocampus was removed for BDNF analysis. Again to control for physically swimming in the pool BDNF was also analysed in matched control animals ( $n=4$ /group).

Finally, to control for whether returning to the testing environment and completion of the retention probe task may somehow 'boost' BDNF expression. Animals $(n=8)$ were trained for 5 days with a subsequent 7-day rest followed by immediate examination of hippocampal BDNF expression (without retention probe task). This was matched by an equivalent group $(n=3)$ that was allowed to swim in the pool for 5 days (without the platform and with timing matched to those trained for 5 days), followed by a week's rest and immediate examination of hippocampal BDNF expression (again without retention probe task).

\subsubsection{ELISA analysis of BDNF expression}

The expression of BDNF in the hippocampal formation was measured via a BDNF Emax $^{\mathrm{TM}}$ ImmunoAssay System (Promega, UK). Hippocampal and EC samples were homogenised on ice, in ice-cold Krebs solution 50 times. Protein was assessed and samples diluted with Krebs solution to give equal protein concentrations, and stored at $-20^{\circ} \mathrm{C}$. Ninety-six-well plates were coated with $100 \mu \mathrm{l}$ anti-BDNF monoclonal antibody diluted (1:1000) in carbonate coating buffer $(0.025 \mathrm{M}$ sodium-bicarbonate, $0.025 \mathrm{M}$ sodium carbonate, $\mathrm{pH}$ 9.7). Plates were covered and incubated overnight at $4{ }^{\circ} \mathrm{C}$. On day two, plates were washed once in Tris- $\mathrm{HCl}$ wash buffer $(20 \mathrm{mM}$ Tris- $\mathrm{HCl}$ (pH 7.6); $150 \mathrm{mM} \mathrm{NaCl} ; 0.05 \%$ Tween $\mathrm{v} / \mathrm{v}$ ) to remove excess antibody. They were then blocked for non-specific binding for $1 \mathrm{~h}$ at room temperature. Post-blocking, plates were washed once before $100 \mu \mathrm{l}$ samples of hippocampal tissue or BDNF standards were added to the wells. Plates were then covered and incubated for $2 \mathrm{~h}$ at room temperature. Aliquots $(100 \mu \mathrm{l})$ of anti-human BDNF pAb (diluted 1:500) were added to the wells, plates were incubated for $2 \mathrm{~h}$ at room temperature and washed 5 times. Aliquots $(100 \mu 1)$ of anti-immunoglobulin Y horseradish peroxidase (1:2000 dilution) were added to the wells and incubated for $1 \mathrm{~h}$ at room temperature. Post-incubation, plates were washed $5 \times$ and $100 \mu l$ of the enzyme substrate (room tem- 
perature TMB One Solution) was added to the wells, and incubated for approximately $15 \mathrm{~min}$ until a blue colour formed in the wells. The reaction was stopped by the addition of $100 \mu \mathrm{l}$ of $1 \mathrm{M}$ hydrochloric acid to the wells. Plates were read at $450 \mathrm{~nm}$ in a 96-well plate reader, and BDNF concentrations were estimated for the standard curve. All data are expressed as mean BDNF $(\mathrm{pg} / 0.1 \mathrm{mg}$ total protein).

\subsection{Results}

\subsubsection{Increase in hippocampal and EC BDNF expression parallels water maze acquisition}

Animals were trained in the MWM for 1,2,3,4 or 5 days $(n=5 /$ group, see Fig. 6a). A $5 \times 2$ mixed factorial ANOVA was used to investigate the expression of BDNF in the hippocampus and entorhinal cortex following this training (Fig. 6b). A main effect for brain area was found $(F(1,20)=286.89, p<.001)$. An interaction effect between brain area and days was also returned $(F(4,20)=14.659$, $p<.001)$. Individual $t$-tests showed the expression of BDNF in both these areas were significantly different on all days (1 Day, $t(8)=10.94, p<.001 ; 2$ Days, $t(8)=7.07, p<.001 ; 3$ Days, $t(8)=16.549, p<.001 ; 4$ Days, $t(8)=13.8, p<.001$; 5 Days, $t(8)=5.594, p<.001$ ) with higher levels found in the hippocampus compared to the entorhinal cortex on each respective day. A main effect for day was also shown overall $(F(4,20)=27.75, p<.001)$ with post-hoc tests showing 5 Days to be significantly different to all other days $(p<.001)$. A further one-way ANOVA found a significant training dependent increase in the levels of BDNF in the hippocampus $(F(4,20)=11.09, p<.001$, black bars $)$. Similarly a one-way ANOVA also showed a significant increase in the levels of BDNF in the entorhinal cortex with increased training $(F(4,20)=11.12, p<.001$, white bars $)$.

In order to disassociate BDNF involvement in physically swimming the MWM to it's involvement in learning the platform's location, we allowed three groups of rats to swim (without a platform) for 1,3 or 5 days $(n=4 /$ group) for equivalent time-matched trials based on the mean for those trained with a platform for 1,3 or 5 days. We found a small but non-significant increase in both hippocampal BDNF expression $(12.5 \pm 1.4, \quad 13.1 \pm 0.7$, $17.87 \pm 2.1 \mathrm{pg} / 0.1 \mathrm{mg}$ total protein for 1,3 and 5 days training respectively $(F(2,12)=2.605, p>.05$, Fig. 6c, black bars) and EC BDNF expression $(2.8 \pm 0.5$, $3.5 \pm 0.7$ and $5.85 \pm 1 \mathrm{pg} / 0.1 \mathrm{mg}$ total protein for the same three groups, $F(2,9)=4.23, p>.05$, Fig. $6 \mathrm{c}$, white bars). A $3 \times 2$ mixed factorial ANOVA demonstrated a main effect for brain area $(F(1,9)=67.665, p<.001)$. Further individual $t$-tests on each day showed differences between the brain areas (1 Day, $t(6)=-6.363, p<.001 ; 3$ Days, $t(6)=-9.694, p<.001 ; 5$ Days, $t(6)=-4.059, p<.001)$ with higher levels indicated in the hippocampal formation on each of these days. A main effect for day was also found overall $(F(2,9)=22.817, p<.001)$ where post-hoc tests showed the levels of BDNF following 5 Days swimming was significantly higher than that of 1 Day $(p<.001)$ and 3 Days $(p<.001)$ of swimming. Importantly, comparisons of hippocampal BDNF expression in trained animals were significantly greater $(F(1,12)=34.823, p<.001)$ than these matched control groups suggesting that BDNF correlates with learning rather than simply swimming in the pool. There was no such difference between EC BDNF expression in the trained animals compared to the match control group. A $2 \times 3$ mixed factorial ANOVA confirmed that neither a main effect for group was found $(F(1,9)=0.092$, $p>.05)$ nor an interaction effect $(F(2,9)=0.348, p>.05)$. A main effect for training duration was shown however $(F(2,9)=11.4, p<.01)$ with 5 Days significantly different to 1 Day $(p<.01)$ and 3 Days $(p<.05)$.

\subsubsection{Learning-related changes in hippocampal BDNF are long lasting}

To examine whether hippocampal BDNF remains expressed over the long-term following acquisition of the water maze task, we trained animals for 1, 2, 3, 4 or 5 days ( $n=4$ /group) and allowed each group seven days rest before a single retention probe trial. As expected, retention of the platform's location (mean percentage time swimming in 'platform area' see Fig. 7a, inset) increased with the amount of training given (grey, squares; $F(4,31)=5.185$, $p<.01$, Fig. 7a). Hippocampal BDNF expression was examined immediately following the retention trial in the groups that had received 1,3 or 5 days training. Fig. 7a (black bars) demonstrate that BDNF expression in the hippocampus increased in line with the amount of training received $(F(2,9)=24.7, p<.001)$. We then compared BDNF expression immediately after training (see above) to the matched trained groups given rest and a retention trial but found no significant difference between the conditions $(F(1,24)=0.592, p>.05)$, suggesting that once expressed BDNF remains relatively increased for at least a week. We also examined whether returning to the testing environment and completion of the retention probe task may somehow 'boost' BDNF expression (Fig. 7b). Animals $(n=8)$ were trained for 5 days with a subsequent 7 day rest followed by immediate examination of hippocampal BDNF expression (without retention) and found a $21 \pm 0.6 \mathrm{pg} /$ $0.1 \mathrm{mg}$ total protein expression of BDNF (significantly decreased in comparison to those with the additional probe trial, $34 \pm 1.9 \mathrm{pg} / 0.1 \mathrm{mg}$ total protein, $t(10)=-8.03$, $p<.01)$. This 'boost' in hippocampal BDNF was also observed in control animals $(n=7)$, that had been allowed to swim for 5 days (without a platform) followed by 7 days rest and returned to the test environment. Hippocampal BDNF expression in this group $(33.1 \pm 1.7 \mathrm{pg} / 0.1 \mathrm{mg}$ total protein) was significantly higher than controls $(n=3)$ that had not returned to the testing environment $(12.57 \pm 0.7 \mathrm{pg} / 0.1 \mathrm{mg}$ total protein, $t(8)=6.83, p<.01$, Fig. 7b). Taken together these results would indicate that hippocampal BDNF increases with spatial training but may decrease in a time-dependent fashion until subsequently boosted by reactivated memory traces. 

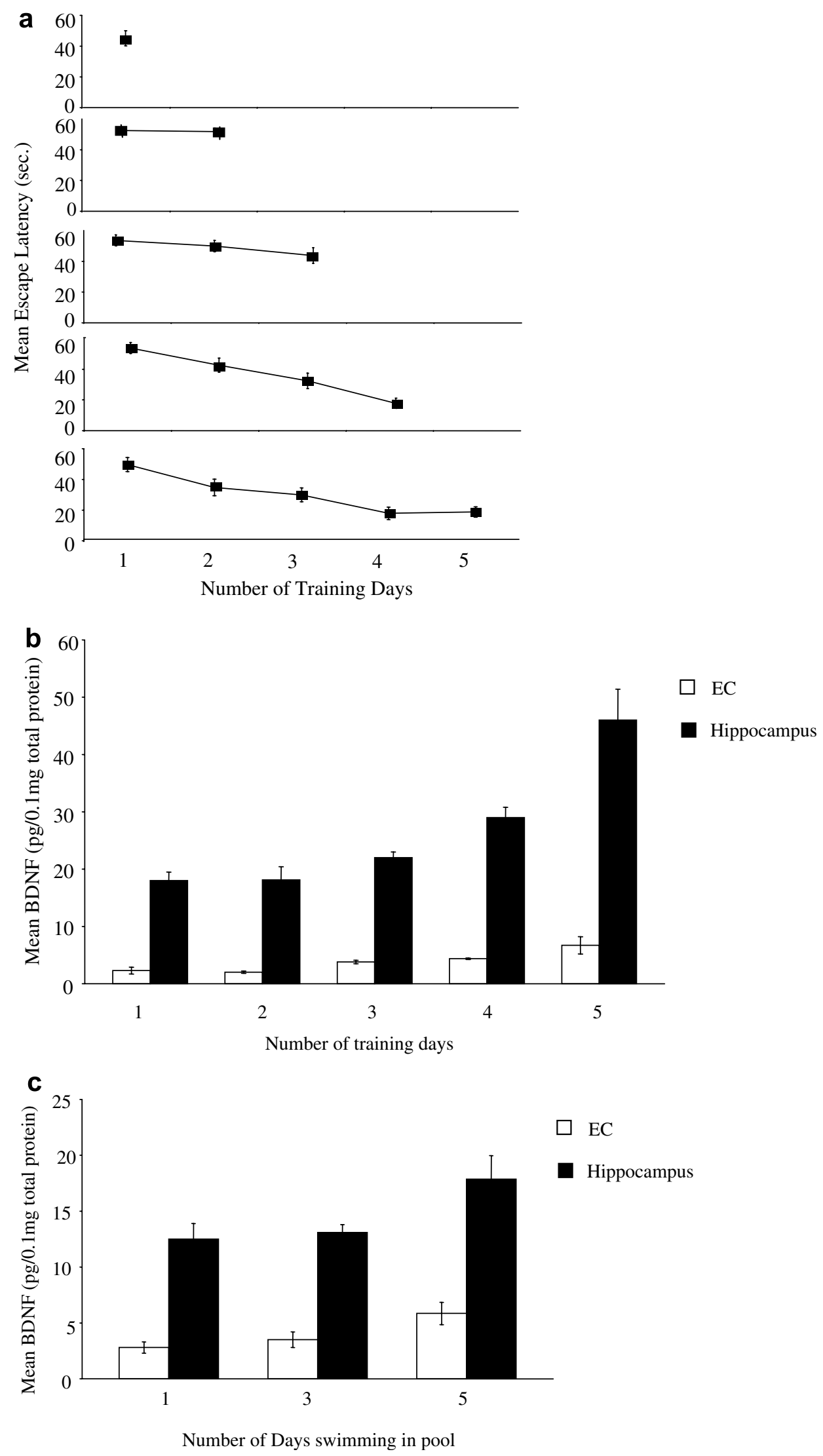

Fig. 6. (a) Mean escape latency in MWM ( $\pm S E M$ ) with (b) mean BDNF (pg/0.1 mg total protein) measured for each training day (Hippocampus (black bars); $18 \pm 1.5,18.13 \pm 2.3,22 \pm 1,29 \pm 1.8$ and $46 \pm 5.4$, EC (white bars); $2.3 \pm 0.6,2.02 \pm 0.2,3.8 \pm 0.3,4.37 \pm 0.13$ and $6.7 \pm 1.5$ for Days $1-5$, respectively). (c) Mean BDNF (pg/0.1 mg total protein) in control animals (no platform present) on days 1, 3 and 5 (Hippocampus; black squares and Entorhinal cortex; white squares). 
a
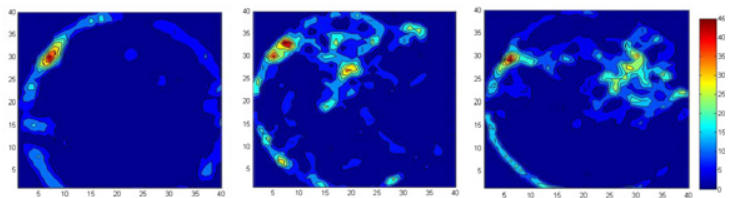

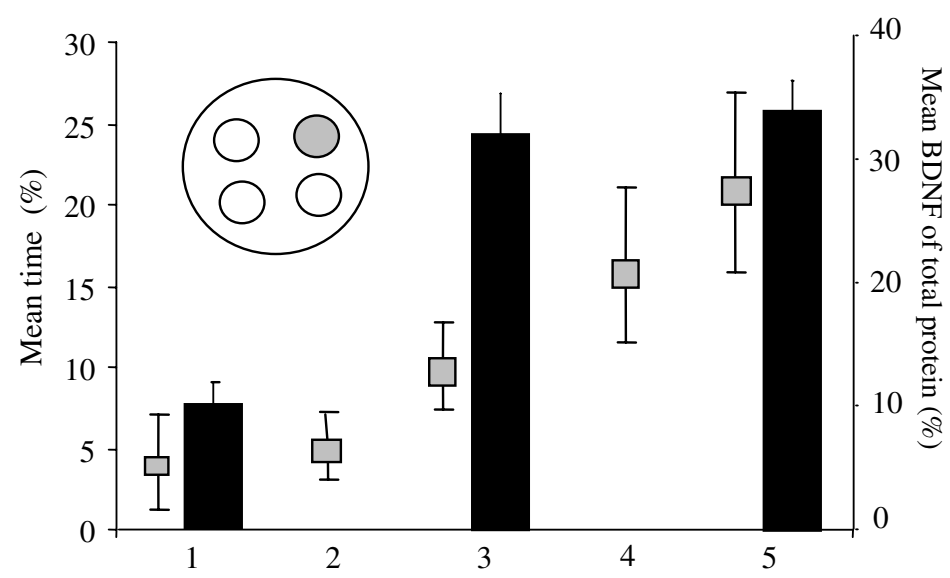

Number of Training Days (with 7 day rest)

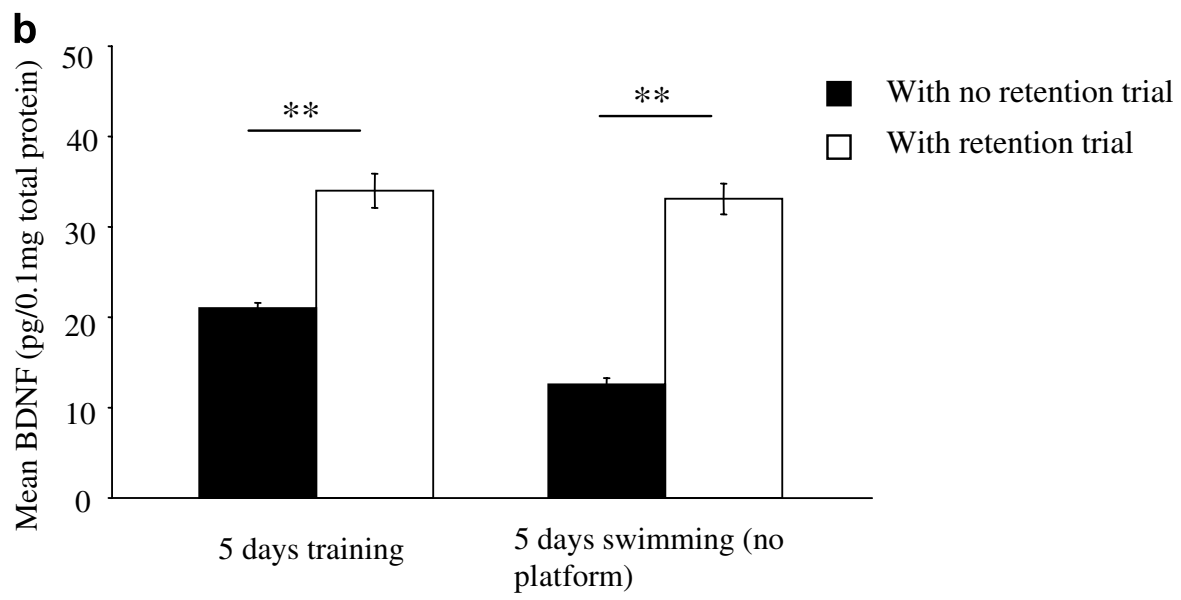

Fig. 7. (a) Mean percentage time in NE 'platform area' (see shaded inset and Section 5.1 ) during the retention trial (grey squares; from $4.0 \pm 2.8 \%$ one training day to $21 \pm 5 \% 5$ training days), with mean hippocampal BDNF (pg/0.1 mg total protein) measured post-retention trial following 1,3 and 5 training days (black bars; $8.5 \pm 1.3,30.9 \pm 3.2$ and $34 \pm 1.9$, respectively) with Matlab density plots showing overall searching activity during retention on these 3 days. (b) Bar chart demonstrating that a return to the water maze for a rention trial "boosted" hippocampal BDNF expression in animals that had been trained for 5 days (left panel) and control animals (right panel).

\section{Discussion}

We suggested in Section 1 that spatial navigation is a dynamic process and that representations are not formed instantaneously in a particular place or time. This idea is confirmed by our analysis of platform behaviour. Preventing cue access while on the platform seemed to have little effect on overall acquisition of the task, when compared to that of animals with unrestricted cue access during the entire task. However, when cues were unavailable during the locomotion phase of the task greater disruptions in overall acquisition emerged (experiment 3 ). These findings would initially suggest that similar to previous research, cue associations with the platform's position are primarily established during in-trial locomotion (Chapillon, 1999;
Devan et al., 1992) and not while on the hidden escape platform (Keith \& Mc Vety, 1988). Alone this result might imply that time on the platform is not required for the formation of spatial relations; however the results of our head-direction platform analysis (experiment 2) show that the mean direction of where an animal looks when on the platform seem to predict their initial heading direction in their subsequent trial, therefore time spent on the platform may contribute somewhat to the overall formation of spatial relations. Not only were animals looking around while on the platform, but additionally their gaze was directed towards the cues on several occasions. Importantly, during the early training days, strong correlations were revealed between the mean direction faced while on the platform and the initial heading direction taken in the pool on the 
following swim trial, strongly suggesting that animals were using a view-matching strategy. While it is accepted that on the early days of training most animals did not 'head' in a particular direction but rather stayed at the side of the pool swimming in a thigmotactic-like fashion, further analysis revealed that the direction in which the animals looked when sitting on the platform also strongly correlated with the mean location of pool-side searching on the subsequent trial. We would predict modelling animal movements at the pool-side (similar to those conducted by Jeanson et al. (2003) in the cockroach (Blattella germanica)) should reveal a non-random pattern of searching during thigmotactic swimming. As training progresses, however, the strength of both sets of correlations show a decrease, signifying that information provided on the platform has a diminishing influence on subsequent searching behaviour, with animals changing from simple view-matching supported by an egocentric-guiding strategy to a more efficient means of escape.

It would appear that during locomotion animals have the ability to assimilate the distal visual cues into their searching of the pool in several ways to form cue-related representations. This is confirmed by the observed use of at least five swimming strategies. Firstly, animals tended to move towards and approach directly the path of a distal cue, and then move along this path for a period of time while holding the cue centrally in their field of vision. This strategy is also often used by other species (e.g. wood ant (Formica rufa; Judd \& Collett, 1998) blowflies (Phaenicia sericata; Campbell, 2001). Judd and Collett (1998), for example, report that when ants approach an object initially they hold the image of this object steady for a period of time on their retina; subsequently when ants revisit the object, they seem to be guided towards the object by matching the current image that forms on the retina to those initial preferred retinal positions. We suggest that animals in the water maze may do something similar; certainly it seems that animals approach a cue and hold this cue for a period of time on their retina. However, as animals are placed into the pool at different locations on subsequent trials we cannot state with certainty that they try to match the current image to those previously remembered. That notwithstanding, we would suggest that by employing this strategy the animals at least gain valuable information on the pool layout and the positions of the cues. As the hidden platform is not located at the landmarks themselves but rather at a distance from them, it is imperative that their cue information is expanded so as to identify where the hidden target is positioned distal to these cues. Therefore, other behaviours which allow for more varied cue interaction are required.

Scanning movement, for example, sees animals move in a constant ambling manner, while still heading in a general unified direction with a cue/s centralised relative to the head. A similar form of egocentric movement is often reported in wasps (Cerceris rybyensis; Jeanson et al., 2003), where it may also act as a method of landmark sampling. We would also suggest that this strategy could be used in an attempt to cover a larger surface area of the pool when searching for the platform, by comparison to approach and stability behaviours, therefore increasing the likelihood of finding the platform. This behaviour, however, was not seen consistently in all animals across days, with a relatively low rate of occurrence throughout acquisition. Further, animals also moved away from the cues and headed towards the pool edge; however the frequency of this behaviour decreased with training, thereby suggesting a maladaptive strategy. As such, by Day 4 or 5 of training animals may have an increased awareness of the importance of the cues or they may simply have an increased overall knowledge of the pool itself. Furthermore, episodes of movement away may also operate as a corrective strategy, in the desert ant (Cataglyphis bicolour) and (Cataglyphis fortis), for example, this behaviour is seen as an active attempt to rectify the discrepancy in their position relative to their earlier stored memory of the landmark (Collett, Dillmann, Giger, \& Wehner, 1992).

It is with the final strategy of "turning" that the use of an allocentric style of navigation appears to be confirmed, particularly in the final days of training. By definition, this strategy represents the use of the distal cues for reorientations during navigation, where animals must 'stop' and verify their position based on their knowledge of the cues spatial relations, and then reorientates on this basis. Not only was turning presented by all animals across each acquisition day with an increase in its frequency seen overall, but the location of turns was also shown to shift in the maze, with a significant decrease in their mean distance relative to the hidden target. We suggest that this highlights the animals increased knowledge of the arena and ability to infer the platform's position using the distal cues. The emergence of this style of navigation in the form of turning behaviour was seen as early as Day 1, but with an increase in its frequency and continual refining of its spatial location shown as training progressed. Indeed, by the final days of training, this strategy was obvious in all animals. Simultaneously, animals also showed evidence of the use of egocentric guidance during their searching of the pool. In a single trial, animals may use an approach and stability strategy, then make a turn followed by another cue guiding strategy (approach and stability or scanning). Therefore animals may actively employ and combine both egocentric-guiding strategies and allocentric inferring strategies in the one trial. While egocentric styles of guidance also emerged on Day 1 and showed some persistence throughout acquisition, their frequency decreased, particularly following Day 3, suggesting this style of navigation was not as dominant as the allocentric alternative. While, previous suggestions have speculated that animals only use one of these strategies singly to successfully locate the platform (e.g. Begega et al., 2001), few have commented on the amalgamation of both within a trial.

Initially we had decided to investigate BDNF as a molecular marker of hippocampal activity, mainly due to its rapid expression in this region soon after learning (Miz- 
uno et al., 2000); what emerges from the current analysis, however, is a clearer understanding of the role that the hippocampal formation may play in spatial navigation, and also the general importance of BDNF in both spatial learning and retention. We demonstrate a significant increase in the expression of BDNF in the hippocampus and entorhinal cortex as training advanced complimenting previous findings by Kesslak et al. (1998) who reported similar increases in hippocampal BDNF following 3 and 6 days of training in the water maze. Although there is strong evidence that exercise can causes increases in BDNF in the brain (Neeper, Gomez-Pinilla, Choi, \& Cotman, 1996), we found increases in hippocampal BDNF that were significantly higher in spatially trained animals compared to those that were not, even though they were exposed to similar testing environment and conditions. This would suggest that, although motor movements may increase BDNF expression, spatial learning enhances hippocampal BDNF further.

Analyses of BDNF concentrations reveal molecular changes in the hippocampal region that, we believe, parallel the observed alternations in the animal's learning behaviour during acquisition. While hippocampal BDNF is expressed on Day 1, its expression increases in a non-linear fashion by Day 5 (somewhat similar to that reported by Kesslak et al., 1998). Many of the behavioural repertoires of the animals change over training and the changes in hippocampal BDNF expression may reflect any of these. So without lesioning the hippocampus and conducting indepth behavioural analysis as described in this article we cannot say for definite how this structure contributes to the way animals solve the MWM task. Although the hippocampus has been shown to be involved in spatial learning generally with evidence that this structure is required for path integration and dead-reckoning (Whishaw, Hines, \& Wallace, 2001) and the formation of allocentric relations in humans (King, Burgess, Hartley, Vargha-Khadem, \& O'Keefe, 2002) and animals (Lavenex, Amaral, \& Lavenex, 2006), we speculate that it is the increase in allocentric inferring abilities that occurs later in the learning period that may be reflected in the observed gradual hippocampal BDNF change. If the hippocampus becomes increasingly active later during the acquisition phase, then this may explain why many authors fail to observe spatial learning deficits despite showing impairments in hippocampal long-term potentiation (LTP; a biologically realistic model for learning and memory, Bliss \& Lomo, 1973). Tyler et al. (2002) for example, suggest that either spatial learning does not require CA1 LTP or the timing of hippocampal LTP may be a critical for spatial learning to occur. We would agree with the second idea, and suggest that in the water maze task hippocampal LTP may occur later during acquisition. Although it is often reported that BDNF plays an important role in the acquisition of hippocampal-dependent behavioural tasks (Mizuno et al., 2000; Yamada et al., 2002), when also examined over a longer time scale (as done in the current study), BDNF levels seem to remain elevated, suggesting this neurotrophin may also play an important role in long-term spatial memory retention. This finding would support previous work suggesting that BDNF may be important for the maintenance phase of LTP (Korte, Kang, Bonhoeffer, \& Schuman, 1998) and may also be necessary for many structural changes at the cellular level. Infusion of BDNF into the dentate gyrus, for example, induces a slow-developing long-lasting potentiation (Messaoudi, Bardsen, Srebro, \& Bramham, 1998), while structurally BDNF has also been shown to enhance dendritic growth and branching (McAllister, Lo, \& Katz, 1995). In addition, there is increasing evidence that BDNF can be recycled by hippocampal neurons and this BDNFinduced BDNF release loop has been strongly implicated in LTP maintenance (Bramham \& Messaoudi, 2005; Santi et al., 2006). It is therefore also possible that the long-lasting enhanced BDNF expression that we have observed in our long-term retention experiments may be a result of a similar BDNF-induced BDNF release type mechanism. Finally, we also found that concentrations of BDNF are 'boosted' by a return to the testing environment, perhaps suggesting a priming of neurotrophic response again perhaps by some neurotrophin-induced neurotrophin release mechanism.

In conclusion, we believe, the present article provides one of the first comprehensive accounts of how the popular MWM task is accomplished behaviourally. Our findings suggest that animals solve the task using distal cues via an initial view-matching strategy that is supported by egocentric guidance. Through increased training, however, the influence of the view-dependent strategy weakens while simultaneously the egocentric-guiding strategy combines with the animal's greater ability to infer the hidden platform's location (via allocentric extrapolation). In addition to this, we also reveal how the hippocampus may contribute to the formation of allocentric representations. As the animal acquires the task, adjustments in behavioural strategies towards a more allocentric-based strategy, are reflected in increased expression of BDNF in the hippocampus and entorhinal cortex. These increases can be directly attributed to spatial learning rather than exercise or other motor influences. Finally, we reveal that hippocampal BDNF can remain increased for at least a week, but can also be 'boosted' by a returning to the testing environment and completion of a retention task, thereby suggesting an additional role for BDNF in spatial learning that has not been previously reported.

\section{Acknowledgments}

The authors gratefully acknowledge the support of Science Foundation Ireland (SFI), Irish Research Council for Science Engineering and Technology (IRCSET), and Irish Research Council for Humanities and Social Sciences (IRCHSS). We also thank Dr. Bernard Mahon (NUIM) for the use of his lab, John Kealy for assistance in data collection, Professors Shane O'Mara (TCD), Drs. Richard 
Roche and Deirdre Desmond (NUIM) for their helpful comments and discussions.

\section{References}

Aggleton, J. P., Keith, A. B., Rawlins, J. N. P., Hunt, P. R., \& Sahgal, A. (1992). Removal of the hippocampus and transection of the fornix produce comparable deficits on delayed non-matching to position by rats. Behavioural Brain Research, 52, 61-71.

Aggleton, J. P., Vann, S. D., Oswald, C. J. P., \& Good, M. (2000). Identifying cortical inputs to the rat hippocampus that subserve allocentric spatial processes: A simple question with a complex answer. Hippocampus, 10, 466-474.

Allen, G. A. (Ed.). (2004). Human spatial memory. New Jersey, Lawrence: Erlbaum Associates, Inc.

Begega, A., Cienfuegos, S., Rubio, S., Santin, J. L., Miranda, R., \& Arias, J. L. (2001). Effects of ageing on allocentric and egocentric spatial strategies in the Wistar rat. Behavioural Processes, 53(1-2), 75-85.

Bekinschtein, P., Cammarota, M., Igaz, L. M., Bevilaqua, L. R., Izquierdo, I., \& Medina, J. H. (2007). Persistence of long-term memory storage requires a late protein synthesis- and BDNF-dependent phase in the hippocampus. Neuron, 53(2), 261-277.

Benhamou, S., \& Poucet, B. (1998). Landmark use by navigating rats (Rattus norvegicus): Contrasting geometric and featural information. Journal of Comparative Psychology, 112, 317-322.

Bliss, T. V., \& Lomo, T. (1973). Long-lasting potentiation of synaptic transmission in the dentate area of the anaesthetized rabbit following stimulation of the perforant path. Journal of Physiology, 232(2), 331-356.

Bramham, C. R., \& Messaoudi, E. (2005). BDNF function in adult synaptic plasticity: The synaptic consolidation hypothesis. Progress in Neurobiology, 76(2), 99-125.

Brown, M. F. (1992). Does a cognitive map guide choices in the radial-arm maze? Journal of experimental psychology. Animal behavior processes, 18(1), 56-66.

Campbell, H. R. (2001). Orientation discrimination independent of retinal matching by blowflies. Journal of Experimental Biology, 204, 15-23.

Chapillon, P. (1999). Very brief exposure to visual distal cues is sufficient for young mice to navigate in the Morris watermaze. Behavioural Processes, 46, 15-24.

Cheng, K. (1986). A purely geometric module in the rat's spatial representation. Cognition, 23, 149-178.

Cimadevilla, J. M., Wesierska, M., Fenton, A. A., \& Bures, J. (2001). Inactivating one hippocampus impairs avoidance of a stable roomdefined place during dissociation of arena cues from room cues by rotation of the arena. Proceedings of the National Academy of Sciences of the United States of America, 98(6), 3531-3536.

Cohen, J., \& Bussey, K. (2003). Rats form cognitive maps from spatial configurations of proximal arm cues in an enclosed 4-arm radial maze. Learning and Motivation, 34, 168-184.

Collett, T. S., Dillmann, E., Giger, A., \& Wehner, R. (1992). Visual landmarks and route following in desert ants. Journal of Comparative Physiology, 170, 434-442.

Commins, S., Gemmell, C., Anderson, M., Gigg, J., \& O'Mara, S. M. (1999). Disorientation combined with bilateral parietal cortex lesions causes path integration deficits in the water maze. Behavioural Brain Research, 104(1-2), 197-200.

de Bruin, J. P. C., Moita, M. P., de Brabander, H. M., \& Jooster, R. (2001). Place response learning of rats in a Morris water maze: Differential effects of fimbria-fornix and medial prefrontal cortex lesions. Neurobiology Learning \& Memory, 75, 164-178.

Devan, B. D., Blank, G. S., \& Petri, H. L. (1992). Place navigation in the Morris water task: Effects of reduced platform interval lighting and pseudorandom platform positioning. Psychobiology, 20, 120-126.

D’Hooge, R., \& De Deyn, P. P. (2001). Applications of the Morris water maze in the study of learning and memory. Brain Research Reviews, 36, 60-90.
Gooney, M., \& Lynch, M. A. (2001). Long-term potentiation in the dentate gyrus of the rat hippocampus is accompanied by brain-derived neurotrophic factor-induced activation of TrkB. Journal of Neurochemistry, 77(5), 1198-1207.

Gooney, M., Shaw, K., Kelly, A., O’Mara, S. M., \& Lynch, M. A. (2002). Long-term potentiation and spatial learning are associated with increased phosphorylation of TrkB and ERK in dentate gyrus: Evidence for a role for BDNF. Behavioural Neuroscience, 116, 455-463.

Hall, J., Thomas, K. L., \& Everitt, B. J. (2000). Rapid and selective induction of BDNF expression in the hippocampus during contextual learning. Nature Neuroscience, 3(6), 533-535.

Hamilton, D. A., Rosenfelt, C. S., \& Whishaw, I. Q. (2004). Sequential control of navigation by locale and taxon cues in the Morris water task. Behavioural Brain Research, 154, 385-397.

Hetherington, P. A., \& Shapiro, M. L. (1997). Hippocampal place fields are altered by the removal of single visual cues in a distance-dependent manner. Behavioural Neuroscience, 111, 20-34.

Hollup, S. A., Kjelstrup, K. G., Hoff, J., Moser, M.-B., \& Moser, E. I. (2001). Impaired Recognition of the goal location during spatial navigation in rats with hippocampal lesions. Journal of Neuroscience, 21(12), 4505-4513.

Jarrard, L. E. (1993). On the role of the hippocampus in learning and memory in the rat. Behavioural Neural Biology, 60, 9-26.

Jeanson, R., Blanco, S., Fournier, R., Deneubourg, J. L., Fourcassie, V., \& Theraulaz, G. (2003). A model of animal movements in a bounded space. Journal of Theoretical Biology, 225(4), 443-451.

Judd, S. P. D., \& Collett, T. S. (1998). Multiple stored views and landmark guidance in ants. Nature, 392, 710-714.

Keith, J. R., \& Mc Vety, K. M. (1988). Latent place learning in a novel environment and the influences of prior training in rats. Psychobiology, $16(2), 146-151$.

Martin, G. M., Walker, K. M., \& Skinner, D. M. (2003). A single unstable visual cue impairs spatial learning in a watermaze. Learning and Motivation, 34, 87-103.

Kesslak, J. P., So, V., Choi, J., Cotman, C. W., \& Gomez-Pinilla, F. (1998). Learning upregulates brain-derived neurotrophic factor messenger ribonucleic acid: A mechanism to facilitate encoding and circuit maintenance? Behavioral Neuroscience, 112, 1012-1019.

King, J. A., Burgess, N., Hartley, T., Vargha-Khadem, F., \& O’Keefe, J. (2002). Human hippocampus and viewpoint dependence in spatial memory. Hippocampus, 12, 811-820.

Korte, M., Kang, H., Bonhoeffer, T., \& Schuman, E. (1998). A role for BDNF in the late-phase of hippocampal long-term potentiation. Neuropharmacology, 37, 553-559.

Lavenex, P. B., Amaral, D. G., \& Lavenex, P. (2006). Hippocampal lesion prevents spatial relational learning in adult macaque monkeys. Journal of Neuroscience, 26, 4546-4558.

Lee, J. L., Everitt, B. J., \& Thomas, K. L. (2004). Independent cellular processes for hippocampal memory consolidation and reconsolidation. Science, 304, 839-843.

Liu, Z., Turner, F., \& Bures, J. (1994). Impairments of place navigation of rats in the Morris water maze by intermittent light is inversely related to the duration of the flash. Neuroscience Letters, 180, 59-62.

McAllister, A. K., Lo, D. C., \& Katz, L. C. (1995). Neurotrophins regulate dendritic growth in developing visual cortex. Neuron, 15(4), 791-803.

McGauran, A.-T., Harvey, D., Cunningham, L., Craig, S., \& Commins, S. (2004). Retention of cue-based associations in the water maze is timedependent and sensitive to disruption by rotating the starting position. Behavioural Brain Research, 151, 255-266.

Messaoudi, E., Bardsen, K., Srebro, B., \& Bramham, C. R. (1998). Acute intrahippocampal infusion of BDNF induces lasting potentiation of synaptic transmission in the rat dentate gyrus. Journal of Neurophysiology, 79(1), 496-499.

Mizuno, M., Yamada, K., Olariu, A., Nawa, H., \& Nabeshima, T. (2000). Involvement of brain-derived neurotrophic factor in spatial memory formation and maintenance in a radial arm maze test in rats. Journal of Neuroscience, 20, 7116-7121. 
Moghaddam, M., \& Bures, J. (1996). Contribution of egocentric spatial memory to place navigation of rats in the Morris water maze. Behavioural Brain Research, 78, 121-129.

Morris, R. G. M. (1981). Spatial localisation does not require the presence of local cues. Learning and Motivation, 12, 239-260.

Morris, R. (1984). Developments of a water-maze procedure for studying spatial learning in the rat. Journal of Neuroscience Methods, 11, 47-60.

Morris, R. G. M., Garrud, P., Rawlins, J. N. P., \& O'Keefe, J. (1982). Place navigation impaired in rats with hippocampal lesions. Nature, 297, 681-683.

Muller, R. U., \& Kubie, J. L. (1987). The effects of changes in the environment on the spatial firing of hippocampal complex-spike cells. Journal of Neuroscience, 7(7), 1951-1968.

Nakazawa, K., McHugh, T. J., Wilson, M. A., \& Tonegawa, S. (2004). NMDA receptors, place cells and hippocampal spatial memory. Nature Review Neuroscience, 5(5), 361-372.

Neeper, S. A., Gomez-Pinilla, F., Choi, J., \& Cotman, C. W. (1996). Physical activity increases mRNA for brain-derived neurotrophic factor and nerve growth factor in rat brain. Brain Research, 726, 49-56.

Pearce, J. M., Roberts, A. D., \& Good, M. (1998). Hippocampal lesions disrupt navigation based on cognitive maps but not heading vectors. Nature, 396, 75-77.
Santi, S., Cappello, S., Riccio, M., Bergami, M., Aicardi, G., Schenk, U., et al. (2006). Hippocampal neurons recycle BDNF for activitydependent secretion and LTP maintenance. EMBO J., 25(18), 4372-4380.

Sutherland, R. J., \& Linggard, R. (1982). Being there: A novel demonstration of latent spatial learning in the rat. Behavioural Neural Biology, 36(2), 103-107.

Tyler, W. J., Alonso, M., Bramham, C. R., \& Pozzo-Miller, L. D. (2002). From acquisition to consolidation: On the role of brain-derived neurotrophic factor signaling in hippocampal-dependent learning. Learning \& Memory, 9(5), 224-237.

Whishaw, I. Q., Hines, D. J., \& Wallace, D. G. (2001). Dead reckoning (path integration) requires the hippocampal formation: Evidence from spontaneous exploration and spatial learning tasks in light (allothetic) and dark (idiothetic) tests. Behavioural Brain Research, 127, 49-69.

Whishaw, I. Q., \& Jarrard, L. E. (1996). Evidence for extrahippocampal involvement in place learning and hippocampal involvement in path integration. Hippocampus, 6, 513-524.

Yamada, K., Mizuno, M., \& Nabeshima, T. (2002). Role for brain-derived neurotrophic factor in learning and memory. Life Science, 70, 735-744.

Zeil, J., Kelber, A., \& Voss, R. (1996). Structure and function of learning flights in bees and wasps. The Journal of Experimental Biology, 199, $245-252$. 